\title{
A COMPACT, GENERALIZED COMPONENT MODE MISTUNING REPRESENTATION FOR MODELING BLADED DISK VIBRATION
}

\author{
Sang-Ho Lim*, Ronnie Bladh ${ }^{\dagger}$, Matthew P. Castanier ${ }^{\ddagger}$, and Christophe Pierre ${ }^{\S}$ \\ Department of Mechanical Engineering \\ The University of Michigan, Ann Arbor, MI 48109-2125
}

\begin{abstract}
$\underline{\text { Abstract }}$
The forced response of a mistuned bladed disk can be significantly amplified compared to that of a tuned bladed disk. Various reduced-order models have been studied to predict the response of mistuned bladed disks. Most of these models have been tested only for simple mistuning cases that may not be realistic for actual systems. In this paper, a new approach to generate a general reduced-order model for a mistuned system is presented. From this general formulation, a compact reduced-order model for small blade mistuning is also derived in which mistuning is projected to tuned-system normal modes using modal participation factors of cantilevered-blade component modes. The presented mistuning projection method can estimate the effects of complicated mistuning easily from measurable modal mistuning values of mistuned blades.
\end{abstract}

\section{Introduction}

A bladed disk consists of a set of disk-blade sectors that are assumed to be identical. In practice, however, there are always small variations in the structural properties of individual blades resulting from manufacturing tolerances, material deviations, and operational wear. These variations are referred to as mistuning. Due to mistuning, the vibratory response of a real bladed disk may be significantly different from that of a nominal (tuned) bladed disk. There has been a significant amount of research on understanding and predicting the dynamic behavior of mistuned bladed disks. Many of these studies have employed lumped parameter models. ${ }^{1-9}$ Although a lumped parameter model can provide a basic understanding of the influence of mistuning, it cannot be used to predict quantitatively the dynamic response of an actual bladed disk.

Copyright (c) 2003 by Sang-Ho Lim. Published by the American Institute of Aeronautics and Astronautics, Inc. with permission.

${ }^{\star}$ Graduate Student Research Assistant

${ }^{\dagger}$ Visiting Research Investigator (Currently: Development Engineer, ALSTOM Power Ltd., Switzerland)

${ }^{\ddagger}$ Associate Research Scientist, Senior Member of AIAA.

$\S$ Professor, Senior Member of AIAA.
Therefore, finite element models of bladed disks have been employed to yield reduced-order models by using component mode synthesis, ${ }^{10-17}$ a receptance technique, ${ }^{18}$ classical modal analysis with mistuning projection, ${ }^{19}$ or the exact relationship between the responses of tuned and mistuned systems. ${ }^{20}$ The major differences among these reduced-order model techniques are the substructuring approach and the mistuning implementation.

In general, the reduced-order models are obtained by substructuring a bladed disk into disk and blade components, except for two recent approaches. ${ }^{19,20}$ These two approaches use tuned-system normal modes without substructuring to obtain a reduced-order model or compute the forced response of a mistuned system. One advantage of avoiding substructuring is that there is no additional error introduced for the responses of a tuned system. Yang and Griffin's approach ${ }^{19}$ is especially notable. Since the number of tuned-system normal modes required is on the order of the number of blades, the size of the model is smaller than those of any other models. Petrov et al. ${ }^{20}$ also developed a unique method to compute the forced response of mistuned systems using the frequency response function matrix, which is obtained from tuned-system normal modes, instead of building a mistuned reduced-order model. But, in order to include aerodynamic coupling effects, tuned responses should be obtained by solving aerodynamic equations and structural dynamic equations at the same time, which is very costly.

The implementation of mistuning is also a very important item when a reduced-order model is evaluated, because a reduced-order model should be able to simulate actual mistuned systems. Castanier et al. ${ }^{13}$ included mistuning in a component-based reduced-order model by varying blade modal stiffnesses that appear explicitly in a synthesized stiffness matrix. Bladh et al. ${ }^{14}$ extended this method by introducing the projection of mistuning to the normal modes of a tuned cantilevered blade that is fixed at the disk-blade interface. This mistuning projection method has great potential for general implementation in reduced-order models. Since only modal stiffness variations are directly employed in a reduced-order model, the implementation of mistuning is quite efficient. In addi- 
tion, because different variation patterns can be used according to the mistuned frequencies of individual modes, a mistuned system can be modeled realistically. Yang and Griffin ${ }^{19}$ also used a method based on a similar mistuning projection. However, since mistuning in physical coordinates is directly projected to tuned system modes, the mistuned mass and stiffness matrices in physical coordinates (which are not measurable) are required to be estimated. Therefore, only the case in which mistuned stiffness matrices are proportional to the nominal matrix was presented. Petrov et al. ${ }^{20}$ used the FRF matrix to compute the forced response of mistuned systems without knowing the natural frequencies and mode shapes of mistuned systems. Still, this new method requires tuned-system normal modes to build a FRF matrix, tuned forced responses, and mistuning in physical coordinates. So, again, mistuned mass and stiffness matrices in physical coordinates need to be estimated. Therefore, in terms of the practical implementation of mistuning, it is clear that the mistuning projection method of Bladh et al. ${ }^{14}$ is useful, except that it is assumed that the mode shapes of mistuned and tuned blades are same and that only stiffness mistuning is present.

In this paper, a general reduced-order model for a mistuned system is formulated. A mistuned system is represented by the full tuned system plus virtual mistuning components, and a hybrid interface method is employed in order to combine them. The mistuning components are composed of only mass and stiffness deviations from the tuned case. All the degrees of freedom in the mistuning components are considered to be interface degrees of freedom. Since no assumption is made about mistuning during the formulation, the resulting general formulation is applicable to any mistuning case, regardless of whether mistuning is small or large.

Most previous research on mistuned systems has been based on an assumption that mistuning is small, which is not necessarily the case. If there is large mistuning, such as fractures at the tip of a blade, or a significant geometric variation, it is necessary to include many more tuned-system modes or tuned component (disk and blade) modes in the reduced-order models. This is due to the fact that the mass or stiffness matrices can be changed significantly, and the mode shapes of a mistuned blade can be completely different from those of a tuned blade. Because of this difficulty, large mistuning has not been previously captured by a reduced-order model (ROM). In this work, the general formulation allows the generation of an accurate ROM with a reasonable size by employing attachment modes of a tuned system. Using the general ROM for a mistuned system, a largely mistuned system can be modeled and studied. Furthermore, intentional mistuning, which may be not small in local areas, can be efficiently studied.
From the resulting general formulations, a reducedorder model for small mistuning is also derived. This new ROM has the same tuned mode basis, and thus the same small number of degrees of freedom (DOF), as that of Yang and Griffin's method. ${ }^{19}$ Blade mistuning is implemented by a mistuning projection approach that was originally developed by Bladh et al. $^{14}$ and has been refined for generalized blade mistuning cases in this paper. By using the mistuned mode shapes and natural frequencies of only a few cantilevered-blade modes, any type of structural mistuning can be accurately modeled in an ROM. This new approach to small-mistuned bladed disks is referred to as a component mode mistuning (CMM) method. In the preceding studies, ${ }^{14-16}$ a bladed disk was substructured into disk and blade components in order to project mistuning to the normal modes of a cantilevered blade. Here, by using modal participation factors of the cantilevered blade normal modes to describe the blade motion in the tuned-system normal modes, the CMM method successfully projects mistuning to cantileveredblade normal modes without requiring a componentbased representation for the full system. Furthermore, by investigating these modal participation factors, just a few dominant cantilevered-blade normal modes can be used for the mistuning projection.

A major advantage of this method is that, even when there is mistuning in only part of the blade such that the individual modal mistuning patterns are different from each other, the influence of mistuning may still be estimated accurately. That is, any arbitrary pattern of mistuning in the physical mass and stiffness matrices can be efficiently and accurately implemented in a compact reduced-order model using modal mistuning values for a few cantilevered-blade modes. This feature is especially useful for the case in which two groups of bladedominated modes of the tuned system are closely spaced.

The CMM method is also convenient in implementing aerodynamic coupling effects. Aerodynamic coefficients are typically calculated by unsteady aerodynamic codes based on a set of cantilevered-blade normal modes in a cyclic assembly, using a complex cyclic coordinate transformation. ${ }^{21}$ Therefore, the modal participation matrix of cantilevered-blade modes, which is used for mistuning projection, can be readily utilized to transform the calculated aerodynamic coefficients from complex cyclic coordinates to tuned-system modal coordinates.

One of the primary contributions of this paper is that a general reduced-order model for a mistuned system is formulated in a systematic manner, regardless of whether mistuning is small or large. Therefore, the effects of various large structural variations can be studied using an ROM. Another contribution is that, for small mistuning, a compact modeling framework for a bladed disk influenced by aerodynamic coupling and general- 
ized small blade mistuning is presented. In particular, by introducing modal participation factors in the description of the blade motion in tuned-system normal modes, aerodynamic coupling and mistuning are easily implemented. Furthermore, it is found that a few dominant cantilevered-blade normal modes can be identified to represent the blade motion of the full system, and the corresponding modal mistuning values are sufficient to represent the influence of real mistuning.

This paper is organized as follows. The general formulation of a reduced-order model for a mistuned system is presented in Section 2. From this general formulation, a CMM representation for small blade mistuning cases is derived in Section 3. In Section 4, the general formulation is validated by comparing the results of the finite element model (FEM) and the ROM for an industrial turbomachinery rotor with a rogue blade which means large mistuning. In section 5, the CMM approach is applied to the test case industrial rotor and validated by comparing CMM results with FEM results. The test cases cover non-proportional mistuning as well as proportional mistuning, and a shrouded rotor is also tested. The conclusions are summarized in Section 6.

\section{General Reduced-Order Model for a Mistuned System}

A mistuned bladed disk is substructured into a tuned bladed disk and virtual components of which mass and stiffness matrices are defined by the difference between the mistuned system and the tuned system, as shown in Fig. 1. These virtual components are called mistuning components. Since the response of a bladed disk is much more sensitive to mistuning in blades than that in the disk, only blade mistuning is considered in this study. However, the proposed substructuring approach can be applied to any mistuned system.

In order to combine a tuned system and mistuning components, a hybrid interface method is employed. A

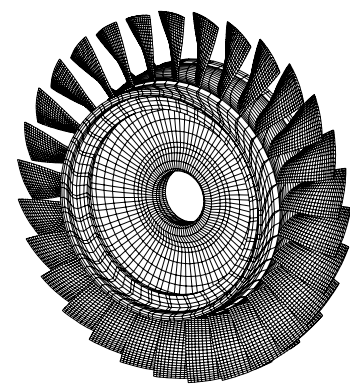

Tuned bladed disk $\left(\mathbf{M}^{\mathrm{S}}, \mathbf{K}^{\mathrm{S}}\right)$

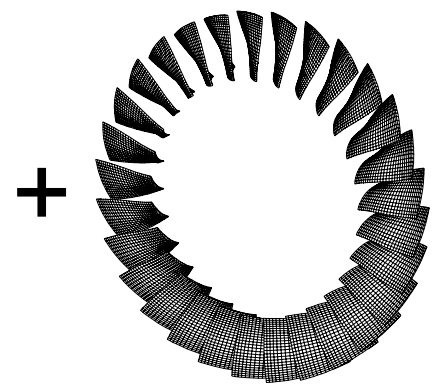

Blade mistuning components $\left(\mathbf{M}^{\delta}, \mathbf{K}^{\delta}\right)$
Fig. 1: Substructuring of a mistuned bladed disk tuned system is treated as a free-interface component, and mistuning components are treated as fixed-interface components. For component mode synthesis (CMS) of the tuned system, normal modes and attachment modes are needed. Attachment modes are obtained by applying a unit force to each interface node. For CMS of a mistuning component, normal modes and constraint modes are needed. The constraint modes are obtained by enforcing a unit displacement at each interface node, successively. Here, it should be noted that, since all the DOF in mistuning components are interface DOF, they do not have component normal modes. Therefore, constraint modes, which become an identity matrix in this case, are enough to describe the motion of mistuning components.

The equations for the free-interface component (a tuned system) can be expressed using its normal modes and attachment modes as follows:

$$
\begin{gathered}
\boldsymbol{\mu}^{\mathrm{S}}=\left[\begin{array}{cc}
\mathbf{I} & \boldsymbol{\Phi}^{\mathrm{S}^{T}} \mathbf{M}^{\mathrm{S}} \mathbf{\Psi}^{\mathrm{S}} \\
\mathbf{\Psi}^{\mathrm{S}^{T}} \mathbf{M}^{\mathrm{S}} \boldsymbol{\Phi}^{\mathrm{S}} & \mathbf{\Psi}^{\mathrm{S}^{T}} \mathbf{M}^{\mathrm{S}} \boldsymbol{\Psi}^{\mathrm{S}}
\end{array}\right] \\
\boldsymbol{\kappa}^{\mathrm{S}}=\left[\begin{array}{cc}
\boldsymbol{\Lambda}^{\mathrm{S}} & \boldsymbol{\Phi}^{\mathrm{S}^{T}} \mathbf{K}^{\mathrm{S}} \boldsymbol{\Psi}^{\mathrm{S}} \\
\mathbf{\Psi}^{\mathrm{S}^{T}} \mathbf{K}^{\mathrm{S}} \boldsymbol{\Phi}^{\mathrm{S}} & \mathbf{\Psi}_{\Gamma}^{\mathrm{S}}
\end{array}\right] \\
\mathbf{x}^{\mathrm{S}}=\left\{\begin{array}{l}
\mathbf{x}_{\Delta}^{\mathrm{S}} \\
\mathbf{x}_{\Gamma}^{\mathrm{S}}
\end{array}\right\}=\left[\begin{array}{ll}
\boldsymbol{\Phi}_{\Delta}^{\mathrm{S}} & \mathbf{\Psi}_{\Delta}^{\mathrm{S}} \\
\boldsymbol{\Phi}_{\Gamma}^{\mathrm{S}} & \mathbf{\Psi}_{\Gamma}^{\mathrm{S}}
\end{array}\right]\left\{\begin{array}{l}
\mathbf{p}_{\phi}^{\mathrm{S}} \\
\mathbf{p}_{\psi}^{\mathrm{S}}
\end{array}\right\},
\end{gathered}
$$

where $\boldsymbol{\mu}^{\mathrm{S}}$ and $\boldsymbol{\kappa}^{\mathrm{S}}$ are reduced mass and stiffness matrices of a tuned system obtained by using a truncated set of normal modes $\left(\boldsymbol{\Phi}^{\mathrm{S}}\right)$ and a complete set of attachment modes $\left(\boldsymbol{\Psi}^{\mathrm{S}}\right), \mathbf{p}_{\phi}^{\mathrm{S}}$ and $\mathbf{p}_{\psi}^{\mathrm{S}}$ are the corresponding modal coordinates, $\Delta$ refers to interior DOF, and $\Gamma$ refers to interface DOF (where mistuning exists).

For mistuning components, the mass and stiffness matrices in modal coordinates are the same as those in physical coordinates.

$$
\begin{gathered}
\boldsymbol{\mu}^{\delta}=\mathbf{I}^{T} \mathbf{M}^{\delta} \mathbf{I}=\mathbf{M}^{\delta} \\
\boldsymbol{\kappa}^{\delta}=\mathbf{I}^{T} \mathbf{K}^{\delta} \mathbf{I}=\mathbf{K}^{\delta} \\
\mathbf{x}^{\delta}=\mathbf{I} \mathbf{p}_{\psi}^{\delta}=\mathbf{p}_{\psi}^{\delta},
\end{gathered}
$$

where $\mathbf{I}$ is an identity matrix representing constraint modes, and $\mathbf{p}_{\psi}^{\delta}$ are the corresponding modal coordinates.

Now, the component assembly is achieved by satisfying displacement compatibility over the component interface (i.e., $\mathbf{x}_{\Gamma}^{\mathrm{S}}=\mathbf{x}^{\delta}$ ), which becomes from Eq. (2) and (4):

$$
\mathbf{x}_{\Gamma}^{\mathrm{S}}=\mathbf{\Phi}_{\Gamma}^{\mathrm{S}} \mathbf{p}_{\phi}^{\mathrm{S}}+\mathbf{\Psi}_{\Gamma}^{\mathrm{S}} \mathbf{p}_{\psi}^{\mathrm{S}}=\mathbf{p}_{\psi}^{\delta}=\mathbf{x}^{\delta} .
$$

This constraint equation leads to the synthesized representation of a mistuned system:

$$
\boldsymbol{\mu}^{\mathrm{syn}}=\boldsymbol{\mu}^{\mathrm{S}}+\left[\begin{array}{ll}
\boldsymbol{\Phi}_{\Gamma}^{\mathrm{S} T} \mathbf{M}^{\delta} \boldsymbol{\Phi}_{\Gamma}^{\mathrm{S}} & \boldsymbol{\Phi}_{\Gamma}^{\mathrm{S}}{ }^{T} \mathbf{M}^{\delta} \boldsymbol{\Psi}_{\Gamma}^{\mathrm{S}} \\
\boldsymbol{\Psi}_{\Gamma}^{\mathrm{S} T} \mathbf{M}^{\delta} \mathbf{\Phi}_{\Gamma}^{\mathrm{S}} & \mathbf{\Psi}_{\Gamma}^{\mathrm{S} T} \mathbf{M}^{\delta} \boldsymbol{\Psi}_{\Gamma}^{\mathrm{S}}
\end{array}\right]
$$




$$
\begin{aligned}
& \boldsymbol{\kappa}^{\mathrm{syn}}=\boldsymbol{\kappa}^{\mathrm{S}}+\left[\begin{array}{ll}
\mathbf{\Phi}_{\Gamma}^{\mathrm{S}}{ }^{T} \mathbf{K}^{\delta} \boldsymbol{\Phi}_{\Gamma}^{\mathrm{S}} & \mathbf{\Phi}_{\Gamma}^{\mathrm{S}}{ }^{T} \mathbf{K}^{\delta} \mathbf{\Psi}_{\Gamma}^{\mathrm{S}} \\
\mathbf{\Psi}_{\Gamma}^{\mathrm{S} T} \mathbf{K}^{\delta} \mathbf{\Phi}_{\Gamma}^{\mathrm{S}} & \mathbf{\Psi}_{\Gamma}^{\mathrm{S} T} \mathbf{K}^{\delta} \mathbf{\Psi}_{\Gamma}^{\mathrm{S}}
\end{array}\right] \\
& \mathbf{p}^{\mathrm{syn}}=\left\{\begin{array}{l}
\mathbf{p}_{\phi}^{\mathrm{S}} \\
\mathbf{p}_{\psi}^{\mathrm{S}}
\end{array}\right\} .
\end{aligned}
$$

As formulated in Eq. (6), a mistuned system is described with only the normal modes and attachment modes of a tuned system. Since no assumption about mistuning has been made in deriving this equation, this general formulation is applicable for all kinds of mistuned systems. The only restriction for this formulation is that the number of DOF in a mistuned part must be the same as that of the corresponding part of a tuned system. The number of attachment modes required is the same as the number of DOF of the mistuning components. Although the number of attachment modes may be huge, the attachment modes improve the convergence rate of the natural frequencies and mode shapes of an ROM as the number of tuned-system normal modes increases, as discussed in section 4 . Therefore, an accurate ROM with a reasonable size can be achieved in a systematic manner by using this general formulation.

\section{Small Mistuning Model}

A general reduced-order model for a mistuned system has been developed in the previous section. In this section, the general ROM is simplified by assuming that mistuning is small comparing to nominal properties in the modal domain, i.e., $\left|k_{i j}^{\delta} / k_{i j}^{\mathrm{S}}\right| \ll 1$. Also, a new method to implement mistuning in the simplified ROM is presented.

For small mistuning, blade-alone natural frequency deviations are usually used to represent mistuning in the ROM. The new method presented in this paper for the implementation of mistuning also employs mistuning values in modal coordinates that can be obtained from natural frequencies and mode shapes. The direct use of modal mistuning values is efficient, especially in Monte Carlo simulations for which a number of mistuning patterns are required as inputs. Since the new method uses mistuning in modal coordinates of component modes of a tuned blade, it is called Component Mode Mistuning (CMM) method in this paper.

\subsection{Approximation by Small Mistuning}

Recently, Yang and Griffin reported on modal interaction $^{22}$ and applied the results to the modeling of mistuned bladed disks. ${ }^{19}$ The main idea for their method is that, when a tuned bladed disk assembly has normal modes closely spaced in a frequency range, a slightly mistuned bladed disk also has normal modes closely spaced in the same frequency range, and thus the mistuned normal modes can be captured by a subset of the tuned nor- mal modes. This means that other tuned normal modes outside of the frequency range of interest, or any static modes, can be ignored in modeling a mistuned system with small mistuning.

Now, if $\boldsymbol{\Psi}^{\mathrm{S}}$ and the corresponding modal coordinates, $\mathbf{p}_{\psi}^{\mathrm{S}}$, in Eq. (6) are ignored, the synthesized representation becomes

$$
\begin{aligned}
& \boldsymbol{\mu}^{\mathrm{syn}}=\left[\mathbf{I}+\boldsymbol{\Phi}_{\Gamma}^{\mathrm{S}}{ }^{T} \mathbf{M}^{\delta} \boldsymbol{\Phi}_{\Gamma}^{\mathrm{S}}\right] \\
& \boldsymbol{\kappa}^{\mathrm{syn}}=\left[\boldsymbol{\Lambda}^{\mathrm{S}}+\boldsymbol{\Phi}_{\Gamma}^{\mathrm{S}}{ }^{T} \mathbf{K}^{\delta} \boldsymbol{\Phi}_{\Gamma}^{\mathrm{S}}\right] \\
& \mathbf{p}^{\mathrm{syn}}=\left\{\mathbf{p}_{\phi}^{\mathrm{S}}\right\},
\end{aligned}
$$

where

$$
\mathbf{M}^{\delta}=\underset{n=1, \ldots, N}{\mathbf{B d i a g}}\left[\mathbf{M}_{n}^{\delta}\right], \quad \mathbf{K}^{\delta}=\underset{n=1, \ldots, N}{\mathbf{B d i a g}}\left[\mathbf{K}_{n}^{\delta}\right],
$$

and $\mathbf{p}_{\phi}^{\mathrm{S}}$ are modal coordinates corresponding to normal modes of the tuned system closely spaced in a frequency range. Bdiag $[\cdot]$ denotes a block-diagonal matrix with the argument being the $n$th block corresponding to the $n$th blade, and $N$ is the number of blades. Since, in most cases, a bladed disk has high modal density in some frequency ranges and the number of normal modes in each of those frequency ranges are on the order of the number of blades, Eq. (7) can represent a small-mistuned bladed disk accurately with matrices of order $N$.

It can be observed in Eq. (7) that mass and stiffness deviation matrices in physical coordinates are projected to the blade portion of tuned-system normal modes. Note that, in this study, only blades are considered to have mistuning. In order to obtain the reduced mass and stiffness matrices, it is required to estimate $\mathbf{M}^{\delta}$ and $\mathbf{K}^{\delta}$ so that their dynamic characteristics can match those of the actual blades in a certain frequency range. However, the estimation becomes infeasible when $\mathbf{M}^{\delta}$ and $\mathbf{K}^{\delta}$ are not proportional to nominal values, and thus each blade mode family has a different mistuning pattern.

\subsection{Component Mode Mistuning (CMM) Projection}

Bladh et al. ${ }^{14}$ introduced a mistuning projection method for an ROM. In this method, mistuned mass and stiffness matrices in physical coordinates are projected to the normal modes of a cantilevered tuned blade which is fixed at its root. Using this method, non-proportional mistuning could be implemented efficiently. However, it was assumed that the mode shapes of tuned and mistuned blades were the same. Since the ROM was generated by substructuring a rotor into a disk and blades, the model size was larger than that of Yang and Griffin's model,${ }^{19}$ which is on the order of the number of blades in the bladed disk.

In this section, the blade portion of tuned-system normal modes in Eq. (7) is represented by the modal participation factors of the component modes of a cantilevered 
tuned blade, and the mistuning projection method is employed without estimating $\mathbf{M}^{\delta}$ and $\mathbf{K}^{\delta}$. And also, it will be shown that the mistuning projection method does not necessarily require the tuned and mistuned mode shapes to be the same.

For this projection, first of all, the modal participation factors need to be obtained for cantilevered-blade component modes that represent the blade motion in tunedsystem modes. If only cantilevered-blade normal modes are used to describe the blade motion, the displacements at the boundaries (e.g., blade-disk boundary, shroud-toshroud boundary) cannot be described. Therefore, additional modes to describe the boundary are required. Here, the additional modes are defined as follows:

$$
\left[\begin{array}{c}
\mathbf{\Psi}^{\mathrm{CB}} \\
\mathbf{I}
\end{array}\right]
$$

where $\Psi^{\mathrm{CB}}$ corresponds to the interior DOF of a cantilevered blade, and $\mathbf{I}$ corresponds to the boundary DOF which are fixed when cantilevered-blade normal modes are obtained. And the number of these boundary modes is the same as the number of the boundary DOF so that all the boundary motion can be described. But, it is not possible to measure the mistuning values corresponding to these additional boundary modes. So, it is proposed that boundary modes are determined to minimize their contribution in projecting mistuning and can finally be ignored. Since mistuning may be random, the nominal mass and stiffness matrices of a blade $\left(\mathbf{M}^{\mathrm{CB}}, \mathbf{K}^{\mathrm{CB}}\right)$ are used in minimizing the contribution of boundary modes. Then, the mass and stiffness projections to the boundary modes become

$$
\begin{aligned}
J_{m}\left(\boldsymbol{\Psi}^{\mathrm{CB}}\right) & =\left[\begin{array}{c}
\mathbf{\Psi}^{\mathrm{CB}} \\
\mathbf{I}
\end{array}\right]^{T}\left[\begin{array}{cc}
\mathbf{M}_{\mathrm{ii}}^{\mathrm{CB}} & \mathbf{M}_{\mathrm{ib}}^{\mathrm{CB}} \\
\mathbf{M}_{\mathrm{ib}}^{\mathrm{CB}} & \mathbf{M}_{\mathrm{bb}}^{\mathrm{CB}}
\end{array}\right]\left[\begin{array}{c}
\mathbf{\Psi}^{\mathrm{CB}} \\
\mathbf{I}
\end{array}\right] \\
J_{k}\left(\boldsymbol{\Psi}^{\mathrm{CB}}\right) & =\left[\begin{array}{c}
\boldsymbol{\Psi}^{\mathrm{CB}} \\
\mathbf{I}
\end{array}\right]^{T}\left[\begin{array}{cc}
\mathbf{K}_{\mathrm{ii}}^{\mathrm{CB}} & \mathbf{K}_{\mathrm{ib}}^{\mathrm{CB}} \\
\mathbf{K}_{\mathrm{ib}}^{\mathrm{CB}} & \mathbf{K}_{\mathrm{bb}}^{\mathrm{CB}}
\end{array}\right]\left[\begin{array}{c}
\mathbf{\Psi}^{\mathrm{CB}} \\
\mathbf{I}
\end{array}\right],
\end{aligned}
$$

where the subscript $\mathrm{i}$ and $\mathrm{b}$ denote the interior DOF and the boundary DOF respectively. Now, by taking the first variation of $J_{m}$ and $J_{k}$ in $\Psi^{\mathrm{CB}}$, the boundary modes to minimize the mass and stiffness projections, $\Psi^{\mathrm{CB}, m}$ and $\Psi^{\mathrm{CB}, k}$, can be obtained from the following equations:

$$
\begin{array}{r}
\mathbf{M}_{\mathrm{ii}}^{\mathrm{CB}} \boldsymbol{\Psi}^{\mathrm{CB}, m}+\mathbf{M}_{\mathrm{ib}}^{\mathrm{CB}}=0 \\
\mathbf{K}_{\mathrm{ii}}^{\mathrm{CB}} \boldsymbol{\Psi}^{\mathrm{CB}, k}+\mathbf{K}_{\mathrm{ib}}^{\mathrm{CB}}=0 .
\end{array}
$$

Since the nominal mass and stiffness matrices are used for the projections, the projections represent kinetic energy and stiffness energy of a tuned blade by the boundary motion. Therefore, the obtained boundary modes are the modes to minimize their contribution in kinetic energy and stiffness energy of a tuned blade. It can also be observed from Eq. (9) that, if no mistuning exists in boundary elements, only $\mathbf{M}_{\mathrm{ii}}^{\mathrm{CB}}$ and $\mathbf{K}_{\mathrm{ii}}^{\mathrm{CB}}$ will be used for Eq. (8) and (9), and the boundary modes will be null matrices and provide no contribution to the mistuning projection. Here, it should be noted that $\Psi^{\mathrm{CB}, k}$ is the set of Craig-Bampton constraint modes of a cantilevered blade.

Now, the blade motion in tuned-system modes is described by cantilevered-blade normal modes and boundary modes as follows:

$$
\boldsymbol{\Phi}_{\Gamma, n}^{\mathrm{S}}=\left\{\begin{array}{l}
{\left[\begin{array}{cc}
\boldsymbol{\Phi}^{\mathrm{CB}} & \mathbf{\Psi}^{\mathrm{CB}, m} \\
\mathbf{0} & \mathbf{I}
\end{array}\right]\left[\begin{array}{c}
\mathbf{q}_{\phi, n}^{\mathrm{CB}, m} \\
\mathbf{q}_{\psi, n}^{\mathrm{CB}}
\end{array}\right]} \\
{\left[\begin{array}{cc}
\boldsymbol{\Phi}^{\mathrm{CB}} & \boldsymbol{\Psi}^{\mathrm{CB}, k} \\
\mathbf{0} & \mathbf{I}
\end{array}\right]\left[\begin{array}{l}
\mathbf{q}_{\phi, n}^{\mathrm{CB}, k} \\
\mathbf{q}_{\psi, n}^{\mathrm{CB}}
\end{array}\right],}
\end{array}\right.
$$

where $\boldsymbol{\Phi}_{\Gamma, n}^{\mathrm{S}}$ is the $n$th blade portion of tuned-system modes and $\mathbf{q}_{\phi, n}^{\mathrm{CB}, m}, \mathbf{q}_{\psi, n}^{\mathrm{CB}}, \mathbf{q}_{\phi, n}^{\mathrm{CB}, k}$, and $\mathbf{q}_{\psi, n}^{\mathrm{CB}}$ are the modal participation factors of cantilevered-blade normal modes and boundary modes for the $n$th blade portion. Therefore, all the blade portion can be expressed as follows:

$$
\boldsymbol{\Phi}_{\Gamma}^{\mathrm{S}}=\left\{\begin{array}{l}
\left(\mathbf{I} \otimes \mathbf{U}^{\mathrm{CB}, m}\right) \mathbf{q}^{\mathrm{CB}, m} \\
\left(\mathbf{I} \otimes \mathbf{U}^{\mathrm{CB}, k}\right) \mathbf{q}^{\mathrm{CB}, k}
\end{array}\right.
$$

where

$$
\begin{array}{rlrl}
\mathbf{U}^{\mathrm{CB}, m} & =\left[\begin{array}{cc}
\boldsymbol{\Phi}^{\mathrm{CB}} & \boldsymbol{\Psi}^{\mathrm{CB}, m} \\
\mathbf{0} & \mathbf{I}
\end{array}\right] & \mathbf{U}^{\mathrm{CB}, k}=\left[\begin{array}{cc}
\boldsymbol{\Phi}^{\mathrm{CB}} & \mathbf{\Psi}^{\mathrm{CB}, k} \\
\mathbf{0} & \mathbf{I}
\end{array}\right] \\
\mathbf{q}^{\mathrm{CB}, m}=\left[\begin{array}{c}
\vdots \\
\mathbf{q}_{\phi, n}^{\mathrm{CB}, m} \\
\mathbf{q}_{\psi, n}^{\mathrm{CB}} \\
\vdots
\end{array}\right] & \mathbf{q}^{\mathrm{CB}, k}=\left[\begin{array}{c}
\vdots \\
\mathbf{q}_{\phi, n}^{\mathrm{CB}, k} \\
\mathbf{q}_{\psi, n}^{\mathrm{CB}} \\
\vdots
\end{array}\right],
\end{array}
$$

and $\otimes$ denotes a Kronecker product. The modal participation factors can be easily calculated because a tuned system is a structure with cyclic symmetry (see Appendix). Inserting Eq. (11) into Eq. (7), the reduced mass and stiffness matrices become

$$
\begin{aligned}
& \boldsymbol{\mu}^{\text {syn }} \\
& =\mathbf{I}+\mathbf{q}^{\mathrm{CB}, m^{T}}\left(\mathbf{I} \otimes \mathbf{U}^{\mathrm{CB}, m^{T}}\right) \mathbf{M}^{\delta}\left(\mathbf{I} \otimes \mathbf{U}^{\mathrm{CB}, m}\right) \mathbf{q}^{\mathrm{CB}, m} \\
& =\mathbf{I}+\underset{n=1, \ldots, N}{\mathbf{B d i a g}}\left[\mathbf{q}_{n}^{\mathrm{CB}, m^{T}} \mathbf{U}^{\mathrm{CB}, m^{T}} \mathbf{M}_{n}^{\delta} \mathbf{U}^{\mathrm{CB}, m} \mathbf{q}_{n}^{\mathrm{CB}, m}\right] \\
& =\mathbf{I}+\underset{n=1, \ldots, N}{\mathbf{B d i a g}}\left[\mathbf{q}_{n}^{\mathrm{CB}, m^{T}}\left[\begin{array}{cc}
\boldsymbol{\mu}_{\phi \phi, n}^{\delta} & \boldsymbol{\mu}_{\phi \psi, n}^{\delta} \\
\boldsymbol{\mu}_{\phi \psi, n}^{\delta} & \boldsymbol{\mu}_{\psi \psi, n}^{\delta}
\end{array}\right] \mathbf{q}_{n}^{\mathrm{CB}, m}\right]
\end{aligned}
$$




$$
\begin{aligned}
& \boldsymbol{\kappa}^{\mathrm{syn}} \\
& =\boldsymbol{\Lambda}^{\mathrm{S}}+\mathbf{q}^{\mathrm{CB}, k}\left(\mathbf{I} \otimes \mathbf{U}^{\mathrm{CB}, k}{ }^{T}\right) \mathbf{K}^{\delta}\left(\mathbf{I} \otimes \mathbf{U}^{\mathrm{CB}, k}\right) \mathbf{q}^{\mathrm{CB}, k} \\
& =\boldsymbol{\Lambda}^{\mathrm{S}}+\underset{n=1, \ldots, N}{\mathbf{B d i a g}}\left[\mathbf{q}_{n}^{\mathrm{CB}, k} \mathbf{U}^{\mathrm{CB}, k} \mathbf{K}_{n}^{\delta} \mathbf{U}^{\mathrm{CB}, k} \mathbf{q}^{\mathrm{CB}, k}\right] \\
& =\boldsymbol{\Lambda}^{\mathrm{S}}+\underset{n=1, \ldots, N}{\mathbf{B d i a g}}\left[\mathbf{q}_{n}^{\mathrm{CB}, k} T\left[\begin{array}{cc}
\boldsymbol{\kappa}_{\phi \phi, n}^{\delta} & \boldsymbol{\kappa}_{\phi \psi, n}^{\delta} \\
\boldsymbol{\kappa}_{\phi \psi, n}^{\delta} & \boldsymbol{\kappa}_{\psi \psi, n}^{\delta}
\end{array}\right] \mathbf{q}^{\mathrm{CB}, k}\right],
\end{aligned}
$$

where

$$
\begin{aligned}
\boldsymbol{\mu}_{\phi \phi, n}^{\delta}= & \boldsymbol{\Phi}^{\mathrm{CB} T} \mathbf{M}_{\mathrm{ii}, n}^{\delta} \boldsymbol{\Phi}^{\mathrm{CB}} \\
\boldsymbol{\mu}_{\phi \psi, n}^{\delta}= & \boldsymbol{\Phi}^{\mathrm{CB} T}\left[\mathbf{M}_{\mathrm{ii}, n}^{\delta} \mathbf{\Psi}^{\mathrm{CB}, m}+\mathbf{M}_{\mathrm{ib}, n}^{\delta}\right] \\
\boldsymbol{\mu}_{\psi \psi, n}^{\delta}= & \boldsymbol{\Psi}^{\mathrm{CB}, m^{T}}\left[\mathbf{M}_{\mathrm{ii}, n}^{\delta} \mathbf{\Psi}^{\mathrm{CB}, m}+\mathbf{M}_{\mathrm{ib}, n}^{\delta}\right] \\
& +\mathbf{M}_{\mathrm{ib}, n}^{\delta}{ }^{T} \mathbf{\Psi}^{\mathrm{CB}, m}+\mathbf{M}_{\mathrm{bb}, n}^{\delta} \\
\boldsymbol{\kappa}_{\phi \phi, n}^{\delta}= & \boldsymbol{\Phi}^{\mathrm{CB}{ }^{T}} \mathbf{K}_{\mathrm{ii}, n}^{\delta} \boldsymbol{\Phi}^{\mathrm{CB}} \\
\boldsymbol{\kappa}_{\phi \psi, n}^{\delta}= & \boldsymbol{\Phi}^{\mathrm{CB}{ }^{T}}\left[\mathbf{K}_{\mathrm{ii}, n}^{\delta} \mathbf{\Psi}^{\mathrm{CB}, k}+\mathbf{K}_{\mathrm{ib}, n}^{\delta}\right] \\
\boldsymbol{\kappa}_{\psi \psi, n}^{\delta}= & \boldsymbol{\Psi}^{\mathrm{CB}, k}{ }^{T}\left[\mathbf{K}_{\mathrm{ii}, n}^{\delta} \mathbf{\Psi}^{\mathrm{CB}, k}+\mathbf{K}_{\mathrm{ib}, n}^{\delta}\right] \\
& +\mathbf{K}_{\mathrm{ib}, n}^{\delta}{ }^{T} \mathbf{\Psi}^{\mathrm{CB}, k}+\mathbf{K}_{\mathrm{bb}, n}^{\delta} .
\end{aligned}
$$

Since $\mathbf{M}_{n}^{\delta}$ and $\mathbf{K}_{n}^{\delta}$ are not necessarily proportional to the nominal matrices, $\mathbf{U}^{\mathrm{CB}, m^{T}} \mathbf{M}_{n}^{\delta} \mathbf{U}^{\mathrm{CB}, m}$ and $\mathbf{U}^{\mathrm{CB}, k}{ }^{T} \mathbf{K}_{n}^{\delta} \mathbf{U}^{\mathrm{CB}, k}$ are full matrices as shown in Eq. (12).

In most cases, only a few modal participation factors (usually, just one in unshrouded rotor cases) are dominant, because the blade motion in a tuned-system normal mode tends to be well correlated to that of a cantileveredblade normal mode. (This will be discussed again in section 5.) Therefore, a small number of mistuning values $\left(\boldsymbol{\mu}_{\phi \phi, n}^{\delta}\right.$ and $\left.\boldsymbol{\kappa}_{\phi \phi, n}^{\delta}\right)$ and factors $\left(\mathbf{q}_{\phi, n}^{\mathrm{CB}, m}\right.$ and $\left.\mathbf{q}_{\phi, n}^{\mathrm{CB}, k}\right)$ corresponding to those dominant modes are sufficient for normal mode partitions. Hence, the size of the matrices of mistuning projected to cantilevered-blade component modes is the number of dominant normal modes plus the number of all the boundary modes. When mistuning is non-proportional, this reduced size of mistuning matrices allows highly efficient Monte Carlo simulation with random mistuning values.

Eq. (12) can be used for any mistuned bladed disk. But, it is still required to know the mistuning values related to the boundary modes, which cannot be measured. Now, suppose that the displacements at the blade structural boundaries in the tuned-system normal modes are very small, so that kinetic energy and strain energy due to the boundary displacements are negligible, or that there is no mistuning in the boundary elements. Then, only the dominant cantilevered-blade normal modes are sufficient to project mistuning without losing accuracy. In that case, the partitions related with boundary modes can be ignored and the reduced mass and stiffness matrices can be approximated as follows:

$$
\begin{aligned}
& \boldsymbol{\mu}^{\mathrm{syn}} \cong \mathbf{I}+\underset{n=1, \ldots, N}{\mathbf{B d i a g}}\left[\mathbf{q}_{\phi, n}^{\mathrm{CB}, m^{T}} \boldsymbol{\mu}_{\phi \phi, n}^{\delta} \mathbf{q}_{\phi, n}^{\mathrm{CB}, m}\right] \\
& \boldsymbol{\kappa}^{\mathrm{syn}} \cong \boldsymbol{\Lambda}^{\mathrm{S}}+\underset{n=1, \ldots, N}{\mathbf{B d i a g}}\left[\mathbf{q}_{\phi, n}^{\mathrm{CB}, k^{T}} \boldsymbol{\kappa}_{\phi \phi, n}^{\delta} \mathbf{q}_{\phi, n}^{\mathrm{CB}, k}\right] .
\end{aligned}
$$

Note that $\boldsymbol{\mu}_{\phi \phi, n}^{\delta}$ and $\boldsymbol{\kappa}_{\phi \phi, n}^{\delta}$ still have off-diagonal terms. For simplicity, if only one cantilevered-blade normal mode is dominant in the description of the blade portion of a tuned-system normal mode, only diagonal terms of $\boldsymbol{\mu}_{\phi \phi, n}^{\delta}$ and $\boldsymbol{\kappa}_{\phi \phi, n}^{\delta}$ have dominant effects in computing the matrix operation. In that case, Eq. (13) becomes

$$
\begin{gathered}
\boldsymbol{\mu}^{\mathrm{syn}} \cong \mathbf{I}+\underset{n=1, \ldots, N}{\mathbf{B d i a g}}\left[\mathbf{q}_{\phi_{\mathbf{R}}, n}^{\mathrm{CB}, m^{T}} \underset{r \in \mathbf{R}}{\operatorname{diag}}\left(\mu_{\phi \phi_{r}, n}^{\delta}\right) \mathbf{q}_{\phi_{\mathbf{R}}, n}^{\mathrm{CB}, m}\right] \\
\boldsymbol{\kappa}^{\mathrm{syn}} \cong \mathbf{\Lambda}^{\mathrm{S}}+\underset{n=1, \ldots, N}{\mathbf{B d i a g}}\left[\mathbf{q}_{\phi_{\mathbf{R}}, n}^{\mathrm{CB},{ }^{T}} \underset{r \in \mathbf{R}}{\operatorname{diag}}\left(\kappa_{\phi \phi_{r}, n}^{\delta}\right) \mathbf{q}_{\phi_{\mathbf{R}}, n}^{\mathrm{CB}, k}\right],
\end{gathered}
$$

where

$$
\begin{aligned}
\mu_{r, \phi \phi, n}^{\delta} & =\Phi_{r}^{\mathrm{CB} T} \mathbf{M}_{\mathrm{ii}, n}^{\delta} \Phi_{r}^{\mathrm{CB}} \\
\kappa_{r, \phi \phi, n}^{\delta} & =\Phi_{r}^{\mathrm{CB} T} \mathbf{K}_{\mathrm{ii}, n}^{\delta} \Phi_{r}^{\mathrm{CB}},
\end{aligned}
$$

and $\mathbf{R}$ is a set of dominant cantilevered-blade normal modes.

Now, the estimation of the modal mistuning values $\left(\boldsymbol{\mu}_{\phi \phi, n}^{\delta}\right.$ and $\left.\boldsymbol{\kappa}_{\phi \phi, n}^{\delta}\right)$ is discussed. These mistuning values can be computed if the natural frequencies and mode shapes of mistuned blades are known and the mode shapes of a tuned and mistuned blades are similar, which is usually the case when mistuning is small. For example, the approximate expression for the diagonal terms of $\boldsymbol{\mu}_{\phi \phi, n}^{\delta}$ and $\boldsymbol{\kappa}_{\phi \phi, n}^{\delta}$ to be used in Eq. (15) is shown below.

When the eigenvalue $\left(\boldsymbol{\Lambda}_{n}^{\mathrm{CB}}\right)$ and mode shapes $\left(\boldsymbol{\Phi}_{n}^{\mathrm{CB}}\right)$ of the $n$th real (mistuned) blade are known, the following two approximations can be written:

$$
\begin{aligned}
\mathbf{I}= & \boldsymbol{\Phi}_{n}^{\mathrm{CB}}{ }^{T}\left(\mathbf{M}_{\mathrm{ii}}^{\mathrm{CB}}+\mathbf{M}_{\mathrm{ii}, n}^{\delta}\right) \boldsymbol{\Phi}_{n}^{\mathrm{CB}} \\
= & \boldsymbol{\Phi}_{n}^{\mathrm{CB}}{ }^{T} \mathbf{M}_{\mathrm{ii}}^{\mathrm{CB}} \boldsymbol{\Phi}_{n}^{\mathrm{CB}}+\mathbf{B}_{n}{ }^{T} \boldsymbol{\Phi}^{\mathrm{CB}}{ }^{T} \mathbf{M}_{\mathrm{ii}, n}^{\delta} \boldsymbol{\Phi}^{\mathrm{CB}} \mathbf{B}_{n}(16) \\
= & \boldsymbol{\Phi}_{n}^{\mathrm{CB} T} \mathbf{M}_{\mathrm{ii}}^{\mathrm{CB}} \boldsymbol{\Phi}_{n}^{\mathrm{CB}}+\mathbf{B}_{n}{ }^{T} \boldsymbol{\mu}_{\phi \phi, n}^{\delta} \mathbf{B}_{n} \\
\boldsymbol{\Lambda}_{n}^{\mathrm{CB}} & =\boldsymbol{\Phi}_{n}^{\mathrm{CB}}\left(\mathbf{K}_{\mathrm{ii}}^{\mathrm{CB}}+\mathbf{K}_{\mathrm{ii}, n}^{\delta}\right) \boldsymbol{\Phi}_{n}^{\mathrm{CB}} \\
& =\boldsymbol{\Phi}_{n}^{\mathrm{CB}} \mathbf{K}_{\mathrm{ii}}^{\mathrm{CB}} \boldsymbol{\Phi}_{n}^{\mathrm{CB}}+\mathbf{B}_{n}{ }^{T} \boldsymbol{\Phi}^{\mathrm{CB}}{ }^{T} \mathbf{K}_{\mathrm{ii}, n}^{\delta} \boldsymbol{\Phi}^{\mathrm{CB}} \mathbf{B}_{n} \\
& =\boldsymbol{\Phi}_{n}^{\mathrm{CB}}{ }^{T} \mathbf{K}_{\mathrm{ii}}^{\mathrm{CB}} \boldsymbol{\Phi}_{n}^{\mathrm{CB}}+\mathbf{B}_{n}{ }^{T} \boldsymbol{\kappa}_{\phi \phi, n}^{\delta} \mathbf{B}_{n},
\end{aligned}
$$

where $\mathbf{B}_{n}$ is a matrix consisting of the modal participation factors of tuned cantilevered-blade normal modes for the $n$th mistuned cantilevered-blade normal mode. From these equations, $\boldsymbol{\mu}_{\phi \phi, n}^{\delta}$ and $\boldsymbol{\kappa}_{\phi \phi, n}^{\delta}$ can be computed 
as follows:

$$
\begin{aligned}
& \boldsymbol{\mu}_{\phi \phi, n}^{\delta} \cong \mathbf{B}_{n}{ }^{T}\left(\mathbf{I}-\boldsymbol{\Phi}_{n}^{\mathrm{CB} T} \mathbf{M}_{\mathrm{ii}}^{\mathrm{CB}} \mathbf{\Phi}_{n}^{\mathrm{CB}}\right) \mathbf{B}_{n}{ }^{+} \\
& \boldsymbol{\kappa}_{\phi \phi, n}^{\delta} \cong \mathbf{B}_{n}{ }^{+T}\left(\boldsymbol{\Lambda}_{n}^{\mathrm{CB}}-\mathbf{\Phi}_{n}^{\mathrm{CB}} \mathbf{K}_{\mathrm{ii}}^{\mathrm{CB}} \mathbf{\Phi}_{n}^{\mathrm{CB}}\right) \mathbf{B}_{n}{ }^{+}
\end{aligned}
$$

where $\mathbf{B}_{n}{ }^{+}$is a generalized inverse of $\mathbf{B}_{n}$.

If only the eigenvalues (or natural frequencies) of mistuned blades are known, mistuning values in modal coordinates $\left(\boldsymbol{\mu}_{\phi \phi, n}^{\delta}\right.$ and $\left.\boldsymbol{\kappa}_{\phi \phi, n}^{\delta}\right)$ cannot be obtained without an additional assumption that the mode shapes of tuned and mistuned blades are the same, i.e., $\boldsymbol{\Phi}_{n}^{\mathrm{CB}}=\mathbf{\Phi}^{\mathrm{CB}}$ and $\mathbf{B}_{n}=\mathbf{I}$. With this assumption, only the diagonal terms exist in $\boldsymbol{\kappa}_{\phi \phi, n}^{\delta}$ and no mistuning for mass is allowed. So, the mistuning values become

$$
\boldsymbol{\kappa}_{\phi \phi, n}^{\delta}=\boldsymbol{\Lambda}_{n}^{\mathrm{CB}}-\boldsymbol{\Lambda}^{\mathrm{CB}}
$$

where $\boldsymbol{\Lambda}^{\mathrm{CB}}$ is consisting of the eigenvalues of a tuned cantilevered blade. This estimation of modal mistuning values can be a good approximation because the mode shapes of tuned and mistuned blades are almost the same when mistuning is very small. Note that these eigenvalue mistuning values are already employed in the study by Bladh et al. ${ }^{14}$

So far, the synthesized mass and stiffness matrices of a reduced-order model have been derived using the CMM technique, as in Eq. (12), (13), and (14), and the method to determine the modal mistuning values from the eigenvalues and mode shapes of mistuned blades has been discussed. In the next section, aerodynamic coupling matrices are discussed.

\subsection{Aerodynamic Coupling}

Typically, aerodynamic coefficients are obtained using unsteady aerodynamic codes based on a set of cantilevered-blade normal modes in a cyclic assembly using a complex cyclic coordinate transformation. ${ }^{21}$ Therefore, using the modal participation factors that can be computed in the same way as in the CMM method, aerodynamic coefficients can be projected to the normal modes of a tuned system.

Aerodynamic coefficients related to mass, $\tilde{\mathbf{A}}^{m}$, obtained by using cantilevered-blade normal modes in complex cyclic coordinates are represented by the aerodynamic coupling mass matrix in the physical coordinates, $\mathbf{A}^{m}$, as follows:

$$
\begin{aligned}
\tilde{\mathbf{A}}^{m} & =\left(\mathbf{I} \otimes \mathbf{\Phi}^{\mathrm{CB}^{T}}\right)\left(\mathbf{E}^{*} \otimes \mathbf{I}\right) \mathbf{A}^{m}(\mathbf{E} \otimes \mathbf{I})\left(\mathbf{I} \otimes \boldsymbol{\Phi}^{\mathrm{CB}}\right) \\
& =\left(\mathbf{E}^{*} \otimes \mathbf{I}\right)\left(\mathbf{I} \otimes \mathbf{\Phi}^{\mathrm{CB}}\right) \mathbf{A}^{m}\left(\mathbf{I} \otimes \boldsymbol{\Phi}^{\mathrm{CB}}\right)(\mathbf{E} \otimes \mathbf{I}),
\end{aligned}
$$

where $\mathbf{E}$ is the complex Fourier matrix. Hence, the aerodynamic coupling mass matrix in the tuned-system modal coordinates, $\boldsymbol{\mu}^{\mathrm{a}}$, becomes

$$
\begin{aligned}
\boldsymbol{\mu}^{\mathrm{a}} & =\boldsymbol{\Phi}_{\Gamma}^{\mathrm{S}}{ }^{T} \mathbf{A}^{m} \boldsymbol{\Phi}_{\Gamma}^{\mathrm{S}} \\
& =\mathbf{q}_{\phi}^{\mathrm{CB}}\left(\mathbf{I} \otimes \boldsymbol{\Phi}^{\mathrm{CB}}{ }^{T}\right) \mathbf{A}^{m}\left(\mathbf{I} \otimes \boldsymbol{\Phi}^{\mathrm{CB}}\right) \mathbf{q}_{\phi}^{\mathrm{CB}} \\
& =\mathbf{q}_{\phi}^{\mathrm{CB}}(\mathbf{E} \otimes \mathbf{I}) \tilde{\mathbf{A}}^{m}\left(\mathbf{E}^{*} \otimes \mathbf{I}\right) \mathbf{q}_{\phi}^{\mathrm{CB}} .
\end{aligned}
$$

In the same way, the aerodynamic coupling stiffness $\left(\boldsymbol{\kappa}^{\mathrm{a}}\right)$ and damping $\left(\mathbf{c}^{\mathrm{a}}\right)$ matrices can be obtained.

Eventually, the equation of motion for a smallmistuned system influenced by aerodynamic coupling can be expressed as

$$
\begin{aligned}
{\left[-\omega^{2}\left(\boldsymbol{\mu}^{\mathrm{syn}}\right.\right.} & \left.+\boldsymbol{\mu}^{\mathrm{a}}\right)+j \omega \mathbf{c}^{\mathrm{a}} \\
& \left.+(1+j \gamma) \boldsymbol{\kappa}^{\mathrm{syn}}+\boldsymbol{\kappa}^{\mathrm{a}}\right] \mathbf{p}_{\phi}^{\mathrm{S}}=\boldsymbol{\Phi}^{\mathrm{S}^{T}} \mathbf{f} .
\end{aligned}
$$

\section{$\underline{\text { 4. Large Mistuning Case Study }}$}

The industrial rotor illustrated in Fig. 1 is used in this study. This rotor is the second stage of a four drum compressor rotor used in an advanced gas turbine application. The rotor features 29 blades. The design is referred to as a blisk, since the blades and disk are machined from a single piece of material. The rotor model is clamped at the ribs located at the outer edge of the disk, which is a rough approximation of boundary conditions due to neighboring stages. The rotor model is constructed with standard linear brick elements (eight-noded solids). There are 126,846 DOF in the full model.

Fig. 2 displays the tuned natural frequencies versus number of nodal diameters for the studied rotor. A finite element model of a sector of the rotor was used to obtain the tuned natural frequencies and normal modes. The nearly horizontal connecting lines correspond to tuned-system normal modes dominated by blade motion, while the slanted connecting lines correspond to tunedsystem normal modes dominated by disk motion. Hence, the horizontal lines can be characterized by a cantilever blade normal mode that dominates the blade motion. For instance, the lowest blade-dominated mode family is dominated by the first flexural bending mode $(1 \mathrm{~F})$, and the second lowest blade-dominated mode family is dominated by the first torsional mode (1T). The characterization of blade-dominated mode families are listed on the right-hand side of horizontal lines in Fig. 2, where S denotes a stripe mode and $\mathrm{R}$ denotes elongation in radial direction.

For this large mistuning case study, only one blade is considered to have large mistuning and all the other blades are tuned. The mistuning is introduced by changing the geometry of the blade, which is called a rogue blade in this paper. It is assumed that the geometry is known and mass density and Young's modulus are constant. Therefore, $\mathbf{M}^{\delta}$ and $\mathbf{K}^{\delta}$, which are required to be 


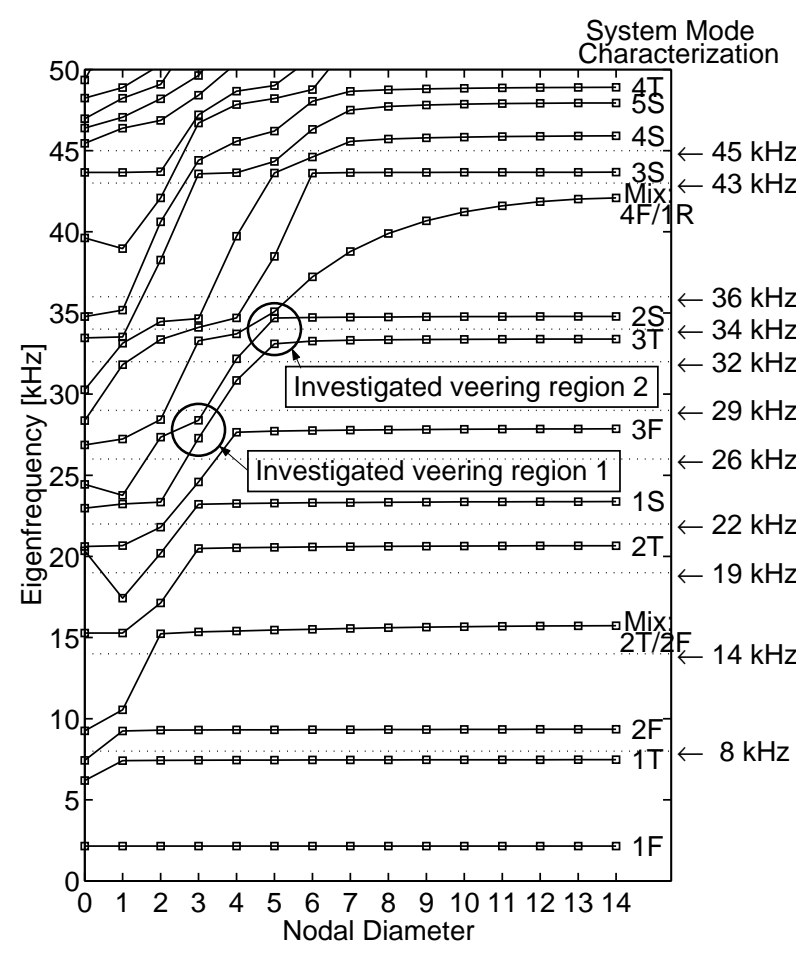

Fig. 2: Natural frequencies versus number of nodal diameters for the unshrouded tuned rotor FEM

known to build a reduced-order model for large mistuning, can be obtained using the geometry. The finite element model of the rogue blade is depicted in Fig. 3. To build a reduced-order model of a rotor with large mistuning, only the one-sector finite element model is sufficient. The attachment modes corresponding to all the DOF of the rogue blade are obtained using cyclic symmetry and the one-sector model.

The size of the resulting reduced-order model is determined by the number of tuned-system normal modes in a truncated set plus the number of attachment modes. For this large mistuning case study, a reduced-order model for the frequency range of $32-36 \mathrm{kHz}$ is built and the results are compared with those of the finite element model of a full mistuned bladed disk. The frequency range for tuned-system normal modes to be employed should be selected to include $32-36 \mathrm{kHz}$. The required attachment modes are those corresponding to the nodes where $\mathbf{M}^{\delta}$ and $\mathbf{K}^{\delta}$ exist. For the test case, all the nodes in the blade are chosen for the attachment modes. Therefore, the model has 66 (tuned normal modes in $32-36 \mathrm{kHz}$ ) +2496 (attachment modes) DOF. Although this size is much greater than that of an ROM for the small mistuning case, it is still a reduced model compared to the full FEM with 126,846 DOF. Once a modal analysis is done for the reduced-order model, 66 normal modes of the mistuned system may be enough to compute the forced

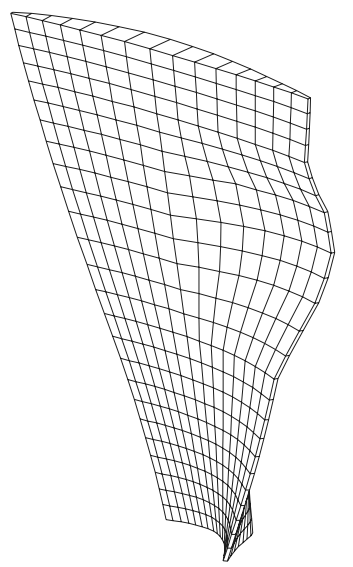

Fig. 3: Rogue blade model

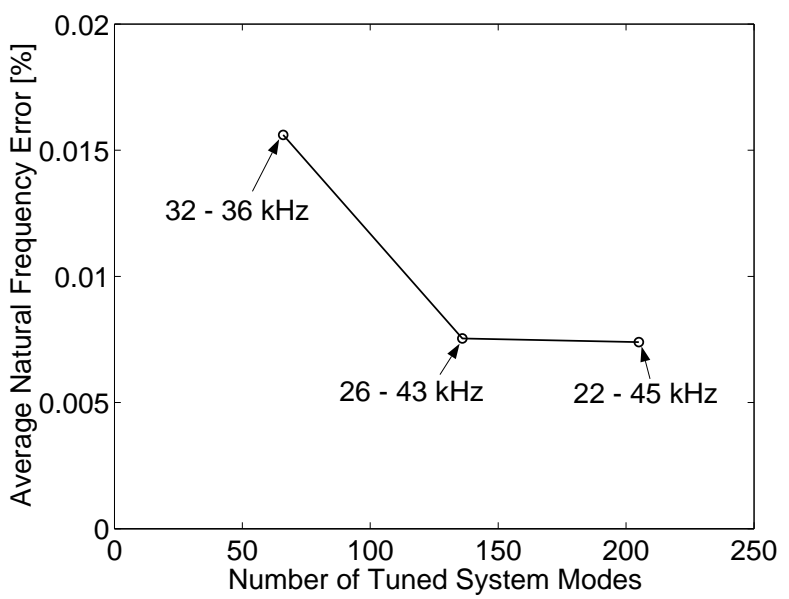

Fig. 4: Convergence of average eigenfrequency error for the largely mistuned rotor

responses for $32-36 \mathrm{kHz}$.

For the validation of the presented method, the convergence of natural frequencies is tested by increasing the number of tuned-system normal modes, and the forced response calculated with the ROM is compared to the FEM results. First, Fig. 4 shows the average natural frequency error versus number of tuned-system normal modes. The tested frequency ranges for the tuned-system normal mode basis are $32-36,26-43$, and $22-45 \mathrm{kHz}$, and the 66 estimated mistuned natural frequencies that exist between 32 and $36 \mathrm{kHz}$ are chosen for comparison with the FEM results. As can be seen in Fig. 4, the estimated natural frequencies converge as the number of tuned-system normal modes increases.

Next, forced responses are discussed. Since, from Fig. 4, it is clear that 136 tuned normal modes in 26-43 $\mathrm{kHz}$ and attachment modes are sufficient to describe the behavior of the mistuned system at $32-36 \mathrm{kHz}, 136$ tuned normal modes and 2496 attachment modes are used to 


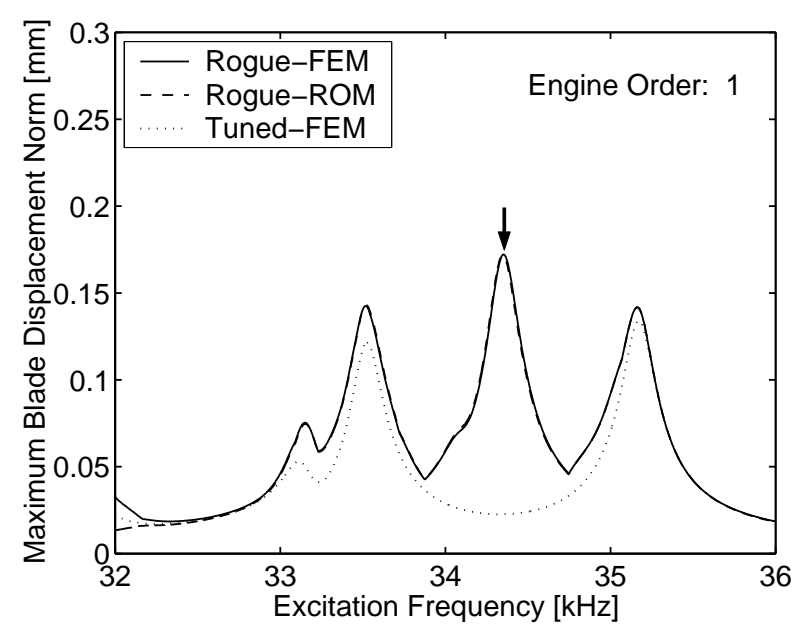

(a)

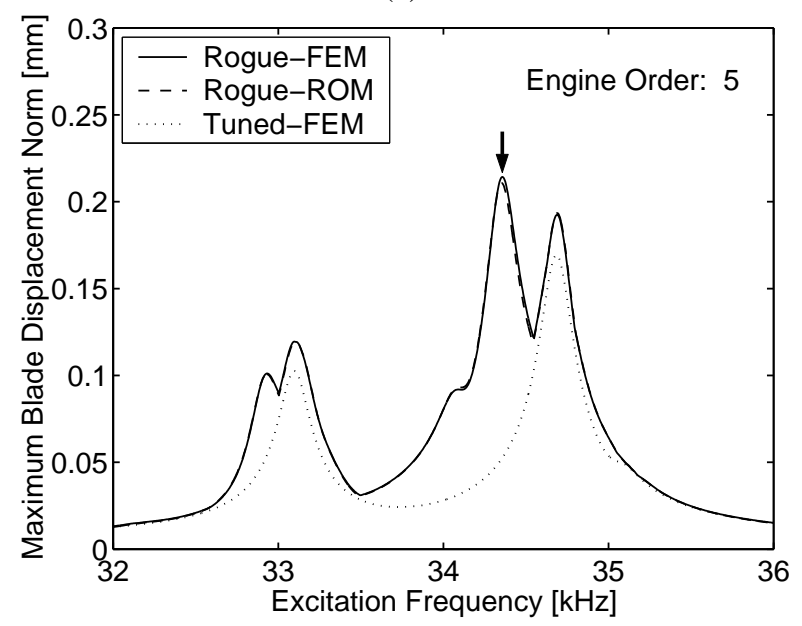

(b)

Fig. 5: Forced responses as obtained by the FEM and an $\operatorname{ROM}(2,632$ DOF) for two cases of engine order excitation

build a reduced-order model. The 66 estimated natural frequencies and mode shapes of the model are chosen as the estimated mistuned modes in the range of 32-36 $\mathrm{kHz}$, in order to compute forced responses. The applied forces are unit loads normal to the blade surface on one of the nodes at the tip of each blade, and engine order 1 and 5 excitations are considered. Euclidean displacement norms for each blade are computed and the maximum value is taken as the amplitude of the forced response at every excitation frequency. The results are shown and compared with FEM results in Fig. 5. As can be seen, the results from the reduced-order model match very well with the results from the FEM.

In Fig. 5, note that one resonant frequency appears around $34.3 \mathrm{kHz}$ in the responses of the mistuned system but not in the responses of the tuned system. The other two largest resonant frequencies are almost the same as

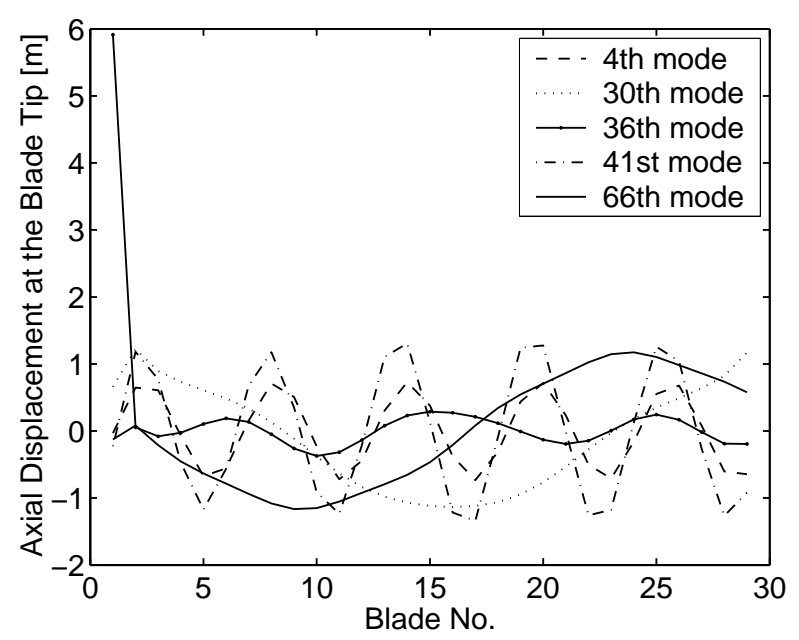

Fig. 6: Mistuned modes: the 4th, 30th, 36th, 41st, and 66th modes of the 66 mistuned modes in the $32-36 \mathrm{kHz}$ range

those of the tuned system. In the case of engine order 1 excitation, the first main peak corresponds to the 30th mode of the 66 mistuned modes in $32-36 \mathrm{kHz}$, the second peak corresponds to the 36th mode, and the third peak corresponds to the 66th mode. In the case of engine order 5 excitation, the three largest peaks correspond to the 4th, 36th mode, and 41st modes. The displacement of a node at the tip of each blade in these modes is depicted in Fig. 6. The lines for the 30th and 66th modes are nearly sinusoidal waves of harmonic 1 along the blade numbers, and the lines for the 4th and 41st modes are nearly sinusoidal waves of harmonic 5. But, the 36th mode is extremely localized to blade 1 , which is the rogue blade. One more thing to note is that the displacement of blade 1 in the 30th, 66th, 4th, and 41st modes is small relative to those of other blades. This is because the natural frequencies and mode shapes of the cantilevered rogue blade are significantly different from the others.

\section{Small Mistuning Case Study}

The CMM method can handle many different kinds of small mistuning in blades. In this paper, for simplicity, mistuning is introduced into the assembly by varying the Young's modulus of blades slightly. That is, only stiffness is mistuned. Young's modulus in a blade is determined as follows:

$$
E_{n}=E_{o}\left(1+\delta_{n}^{E}\right)
$$

where $E_{o}$ is nominal Young's modulus, $E_{n}$ is the Young's modulus of the $n$th blade, and $\delta_{n}^{E}$ is nondimensional mistuning parameter for the Young's modulus of the $n$th blade. If $\delta_{n}^{E}$ is constant in a blade, which 


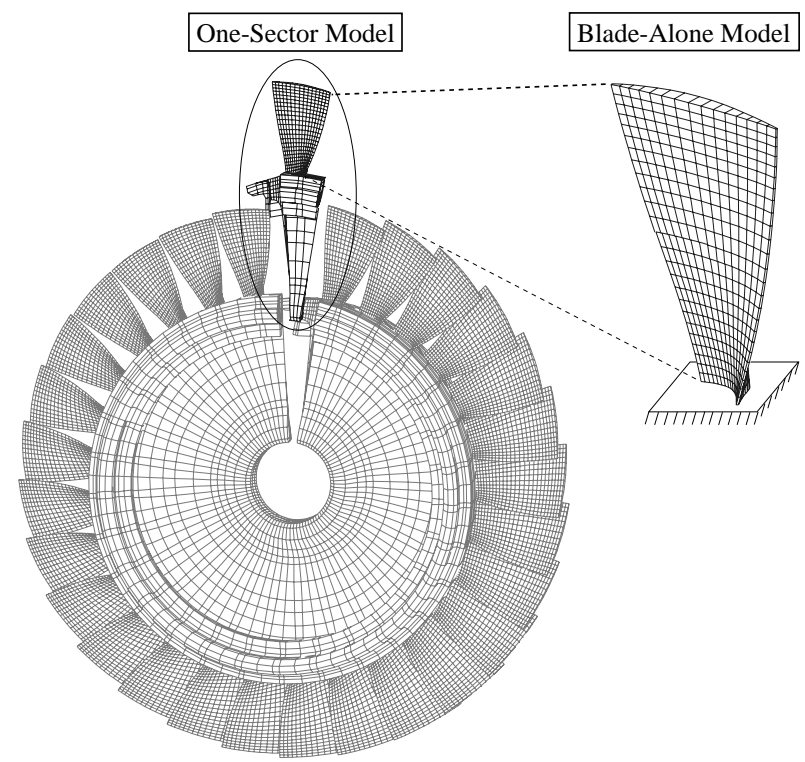

Fig. 7: One-sector model and blade-alone model of an industrial rotor

means proportional mistuning, the natural frequencies of all the cantilevered-blade normal modes will deviate in same percentage and the mode shapes will remain same. Otherwise, the natural frequency deviation patterns will be different for each mode type, and the associated mode shapes will change.

To build a CMM-based reduced-order model of the rotor, the following two tuned finite element models are required, as depicted in Fig. 7:

1. A one-sector model from which the normal modes of the tuned system are obtained via cyclic symmetry analysis. (Commercial FEA software packages, such as MSC/NASTRAN, have cyclic symmetry routines.) There are 4,374 DOF per sector in the finite element model.

2. A blade-alone model from which the cantileveredblade normal modes and the static boundary modes are obtained. This model has a total of 2,496 DOF, and there are 96 DOF at the interface between the blade and the disk.

From the one-sector model, the tuned natural frequencies as well as the tuned mode shapes were obtained. As can be seen in Fig. 2, numerous eigenfrequency veering regions exist. Earlier studies ${ }^{11,23}$ have shown that maximum amplitudes of mistuned forced responses are likely to occur in veering regions. Therefore, the ability to capture mistuned modes and natural frequencies corresponding to veering regions is one of the most important factors for assessing the performance of a modeling method. In order to validate reduced models obtained by the CMM method, the natural frequencies, mode shapes and forced responses by the CMM method are compared with those by a full finite element model. Two eigenfrequency veering regions are investigated: one is located at three nodal diameters, around $28 \mathrm{kHz}$ (region1); the other is located at five nodal diameters, around $34 \mathrm{kHz}$ (region 2). These veering regions are labeled in Fig. 2.

For the studied rotor, the displacements at the blade roots are very small compared to those at the blade interior parts, such that the contribution to strain energy by boundary modes defined at the blade-disk boundary can be neglected. So, normal mode mistuning projection is sufficient for a mistuned reduced-order model. In this section, the CMM technique is validated and the effect of proportional and non-proportional mistuning on the free and forced responses of reduced-order models is investigated using this rotor model. In addition, with the rotor modified to have shrouds, structural coupling and boundary mode mistuning are discussed.

\subsection{Proportional Mistuning}

For the validation of the CMM technique, a mistuned Young's modulus value is introduced for each individual blade. Therefore, the percentage deviations of the natural frequencies of the cantilevered blade are the same for all modes, and the mode shapes of the tuned and mistuned blades are the same. In this case, there are no off-diagonal terms in $\kappa_{\phi \phi, n}^{\delta}$, and only the eigenvalues of the cantilevered blades are mistuned. So, Eq.(14) is appropriate for implementing mistuning and $\boldsymbol{\kappa}_{\phi \phi, n}^{\delta}$ can be computed using Eq. (18). Now, a nondimensional eigenvalue mistuning parameter, $\delta_{r, n}^{e}$, is introduced, which is defined as

$$
\delta_{r, n}^{e}=\frac{\omega_{r, n}^{2}-\omega_{r}^{2}}{\omega_{r}^{2}}
$$

where $\omega_{r, n}$ is the $r$ th natural frequency of the $n$th blade and $\omega_{r}$ is the $r$ th natural frequency of a tuned blade. When proportional stiffness mistuning is introduced, $\delta_{r, n}^{e}$ is the same for any mode $r$, and the eigenvalue mistuning pattern will follow the pattern of the deviations of Young's modulus along the blades. That is, there is only one eigenvalue mistuning pattern.

A specific mistuning pattern for eigenvalues is used to obtain the FEM and CMM results. Table 1 shows the eigenvalue mistuning parameters and the corresponding blades.

The modeling method reported in this study starts from selecting a set of tuned-system normal modes to capture mistuned-system normal modes, and selecting a set of cantilevered-blade normal modes to describe the blade motion in the tuned-system normal modes. Since the modal density is high in the investigated regions in Fig. 2, a narrow frequency band can be chosen for a 


\begin{tabular}{c|r||c|c}
\hline Blade & \multicolumn{1}{|c||}{$\delta_{n}^{e}$} & Blade & \multicolumn{1}{c}{$\delta_{n}^{e}$} \\
\hline 1 & 0.05704 & 16 & 0.04934 \\
2 & 0.01207 & 17 & 0.04479 \\
3 & 0.04670 & 18 & 0.03030 \\
4 & -0.01502 & 19 & 0.00242 \\
5 & 0.05969 & 20 & 0.01734 \\
6 & -0.03324 & 21 & 0.02919 \\
7 & -0.00078 & 22 & -0.00328 \\
8 & -0.01688 & 23 & 0.00086 \\
9 & 0.00242 & 24 & -0.03654 \\
10 & -0.02747 & 25 & -0.03631 \\
11 & -0.03631 & 26 & -0.01665 \\
12 & -0.03570 & 27 & 0.00783 \\
13 & -0.03631 & 28 & -0.01169 \\
14 & -0.03631 & 29 & -0.01332 \\
15 & 0.00242 & & \\
\hline
\end{tabular}

Table 1: Eigenvalue mistuning pattern for the case study rotor with proportional mistuning

tuned-system normal mode basis. For example, 26-29 $\mathrm{kHz}$ can be chosen for veering region 1 . The selection of cantilevered-blade normal modes depends on the tunedsystem normal modes chosen for a basis. Next, modal participation factors need to be calculated using a sufficient number of cantilevered-blade normal modes. And then, by inspecting those factors, dominant cantileveredblade normal modes can be determined.

When modal participation factors for stiffness mistuning are inspected, it should be noted that $\boldsymbol{\kappa}_{\phi \phi, n}^{\delta}$ is pre-multiplied and post-multiplied by the corresponding modal participation factors in the mistuning projection. Therefore, even if a modal participation factor is large, it may be ignored if the corresponding natural frequency of a tuned cantilevered blade is small. Figure 8 shows the average modal participation factors multiplied by the corresponding cantilevered-blade natural frequencies versus cantilevered-blade normal modes when the lowest 30 cantilevered-blade normal modes are used to describe the blade motion in the tuned modes in the ranges 26-29 $\mathrm{kHz}, 32-36 \mathrm{kHz}, 14-45 \mathrm{kHz}$, and $0-50 \mathrm{kHz}$.

It should be noted that the number of cantileveredblade normal modes and the corresponding mistuning patterns do not affect the size of a reduced-order model, but they can affect the accuracy. Nevertheless, a small number of mistuning values are desirable. From Fig. 8, dominant cantilevered-blade normal modes required for accurate mistuning representation can be determined. For instance, only the 7th cantilevered-blade normal mode is dominant for the range of $26-29 \mathrm{kHz}$, because the weighted average modal participation factor of the 7 th mode is much greater than the others. For the same reason, the 8th and 9th modes are dominant for the fre-

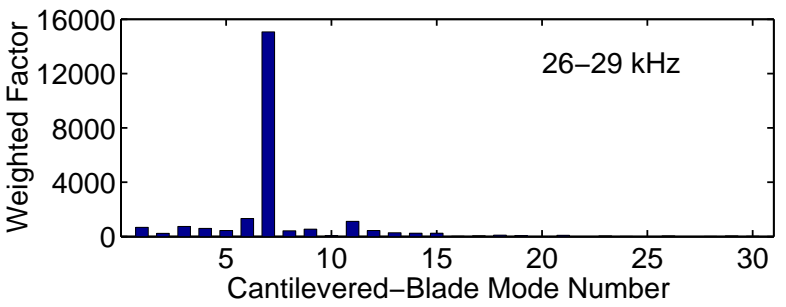

(a)

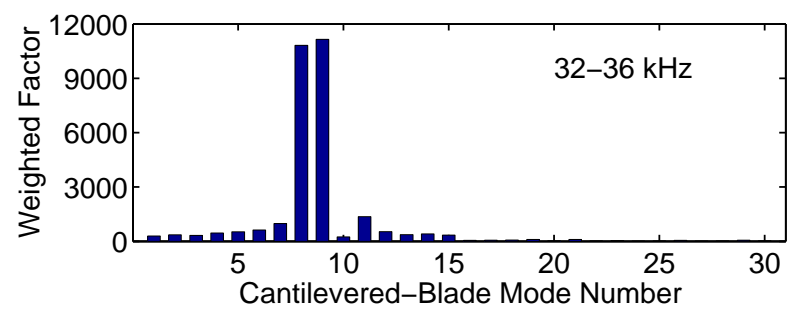

(b)

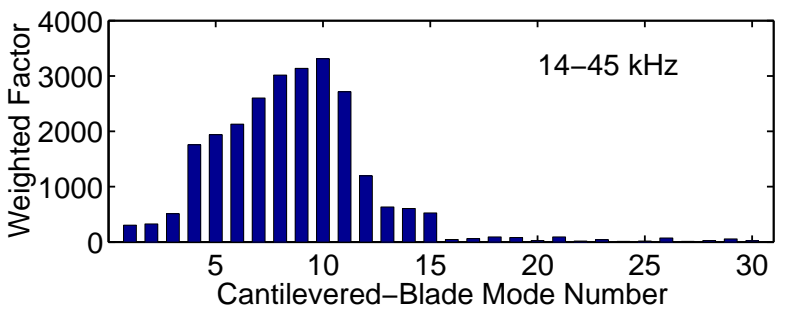

(c)

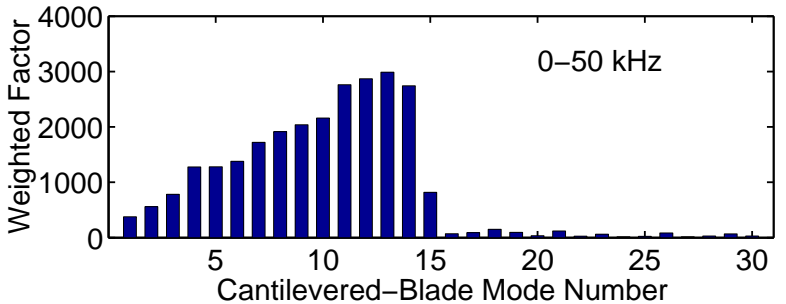

(d)

Fig. 8: Weighted average cantilevered-blade modal participation factor for the blade motion in the tuned-system normal modes in different frequency ranges

quency band of $32-36 \mathrm{kHz}$. This means that the eigenvalue mistuning patterns of the 7th normal mode or the 8th and 9th normal modes of cantilevered blades are enough to predict the behavior of the mistuned system at the veering region 1 or 2 . It is also observed from Fig. 8 that the number of dominant cantilevered-blade normal modes increases as the frequency band for a tuned-system normal mode basis become wide.

Once tuned-system normal modes for a basis and dominant cantilevered-blade normal modes for mistuning projection are chosen, a reduced-order model by the CMM technique can be built with the mistuning values shown in Table 1 . Now, the results from the reducedorder model are compared with those from the FEM. 


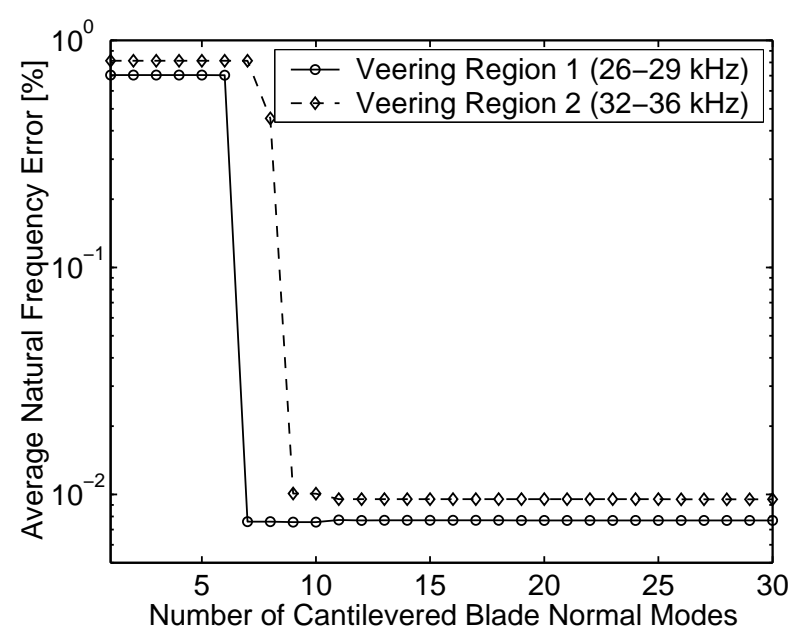

Fig. 9: Convergence of average eigenfrequency error

The convergences of mistuned natural frequencies and MAC (mode assurance criteria) ratios are presented in Fig. 9 and Fig. 10 for two frequency bands: $26-29 \mathrm{kHz}$ for the investigated region 1 and $32-36 \mathrm{kHz}$ for the investigated region 2. Figure 9 shows the average error of the mistuned-system natural frequencies estimated by the CMM models, relative to the natural frequencies by the FEM, versus number of retained cantilevered-blade normal modes, starting from the lowest mode. Figure 10 shows the average MAC ratio between the estimated mistuned modes and the FEM mistuned modes versus number of retained cantilevered-blade normal modes. Since most tuned-system normal modes in the range of 26-29 $\mathrm{kHz}$ are dominated by the 7 th cantilevered-blade normal mode $(3 \mathrm{~F})$, the frequency error and MAC ratio significantly improve when the 7th cantilevered-blade normal mode is employed in the CMM model. Similarly, the frequency error and MAC ratio for the range of 32$36 \mathrm{kHz}$ show great improvement when the 8th and 9th cantilevered-blade normal modes are employed. These convergence trends could be predicted from Fig. 8 (a) and (b).

For the study of forced responses, engine order excitation is considered in the two veering regions indicated in Fig. 2. For veering region 1, engine order 3 excitation is applied in the frequency range of $26-29 \mathrm{kHz}$, and for veering region 2 , engine order 5 and 24 excitations are applied in the frequency range of $32-36 \mathrm{kHz}$. In both cases, the applied forces are unit loads which are the same as those for the large mistuning case study in section 4. The structural damping coefficient is taken to be 0.006 , and the effect of aerodynamic coupling is neglected for simplicity.

The resulting tuned and mistuned forced responses of veering region 1 are shown in Fig. 11 and 12. For the re-

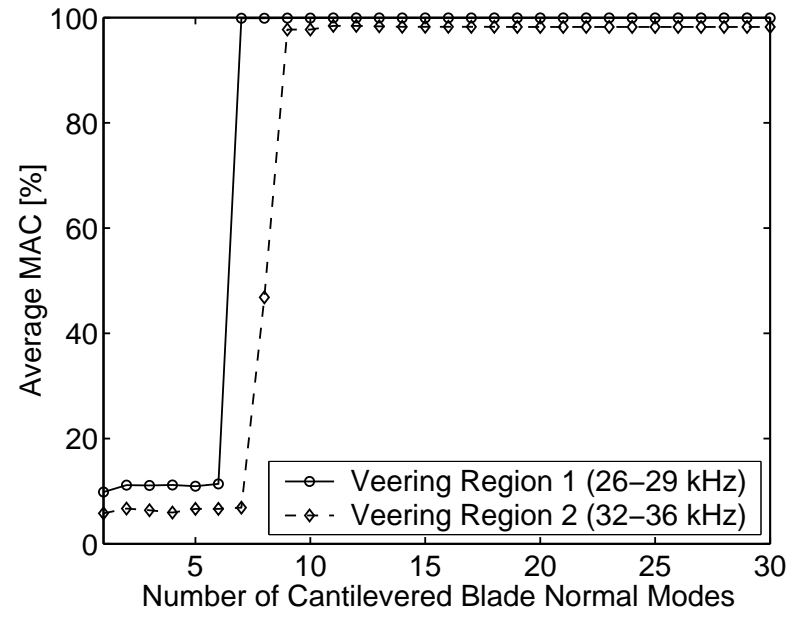

Fig. 10: Convergence of average MAC ratio

sult depicted in Fig. 11, 34 tuned-system normal modes $(26-29 \mathrm{kHz})$ are used to capture mistuned-system normal modes. Figure 12 provides the result when 106 tuned-system modes $(22-34 \mathrm{kHz})$ are selected. In the case of 34 tuned-system normal modes, only the 7th cantilevered-blade normal mode is employed to project mistuning to the tuned-system normal modes. In the case of 106 tuned-system normal modes, the 6th, 7th, and 8th cantilevered-blade normal modes are used because the frequency range for the tuned-system mode basis is 22$34 \mathrm{kHz}$. Note that the maximum amplitude of the mistuned forced responses is 2.24 times larger than that of the tuned forced responses. In Fig. 11, one can see slight differences between the FEM results and CMM results for both the tuned and mistuned responses, although the average natural frequency error is $0.0075 \%$ and the average MAC ratio is $99.8958 \%$ for this reduced model. This difference can be explained. All the mistuned-system natural frequencies and mode shapes estimated by the CMM model are in the range of $26-29 \mathrm{kHz}$. Hence, the effect of modes out of this frequency range is not included, and there exists a difference between CMM results and FEM results even for tuned responses. As can be seen in Fig. 12, when a wider frequency band is chosen, the discrepancy between FEM results and CMM results decreases. But, considering only peak amplitudes at resonant frequencies, the 34-DOF CMM model results match FEM results with negligible errors.

Veering region 2 is more complicated in terms of two close blade-dominated mode families retained in the corresponding frequency range. A group of 66 tuned-system modes $(32-36 \mathrm{kHz})$ are used for the CMM modeling, and the 8th (3T) and 9th (2S) cantilevered-blade normal modes are used for the mistuning projection. Figures 13 and 14 depict the force responses when engine order 5 


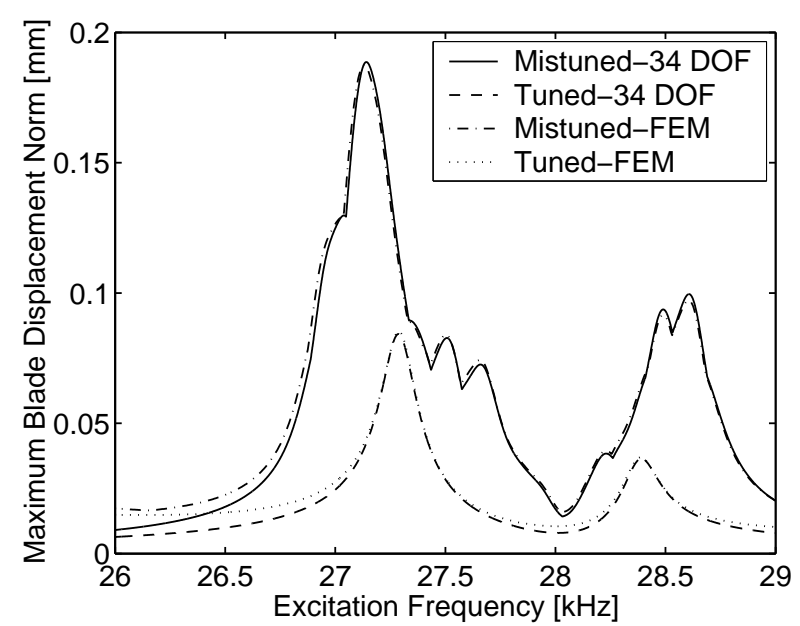

Fig. 11: Forced response for engine order 3 excitation, as obtained by the FEM and a 34-DOF CMM model

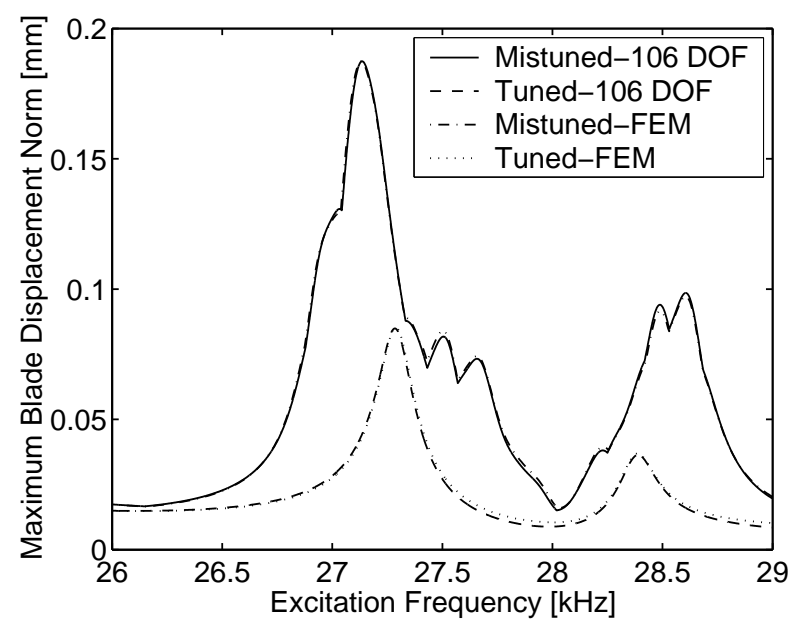

Fig. 12: Forced response for engine order 3 excitation, as obtained by the FEM and a 106-DOF CMM model

and 24 excitations are applied. These figures show excellent matches between CMM results and FEM results, and that the effect of modes outside of $32-36 \mathrm{kHz}$ is negligible. Note that, since the difference between engine order 5 excitation and engine order 24 excitation is only the sign of the phase angle of the force vector, the forced responses for the tuned system in both cases are same. But, for a mistuned system, the forced responses are different. The amplification factors of the maximum forced response amplitude are 1.51 for engine order 5 excitation and 1.34 for engine order 24 excitation.

\subsection{Non-proportional Mistuning}

In this section, non-proportional mistuning is considered. That is, mistuned blades have different eigenvalue mistuning patterns for different cantilevered-blade nor-

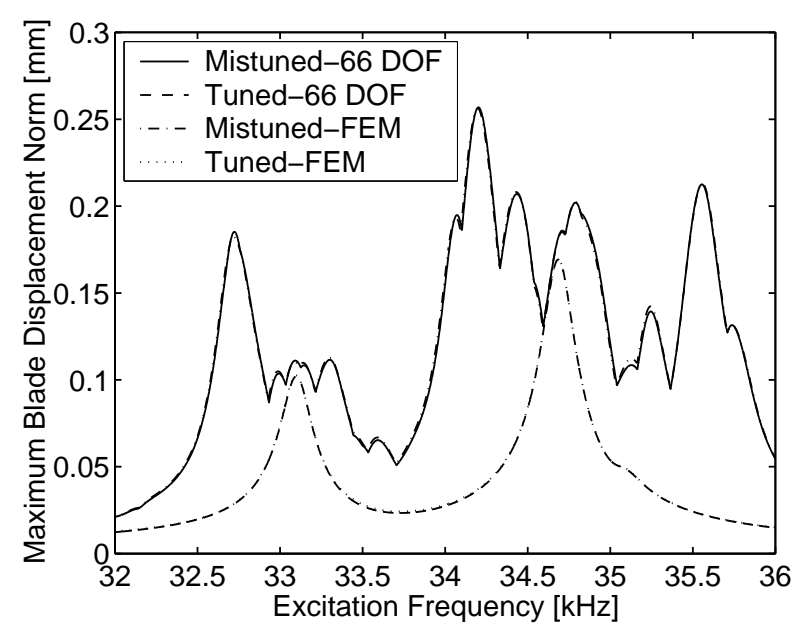

Fig. 13: Forced response for engine order 5 excitation, as obtained by the FEM and a 66-DOF CMM model

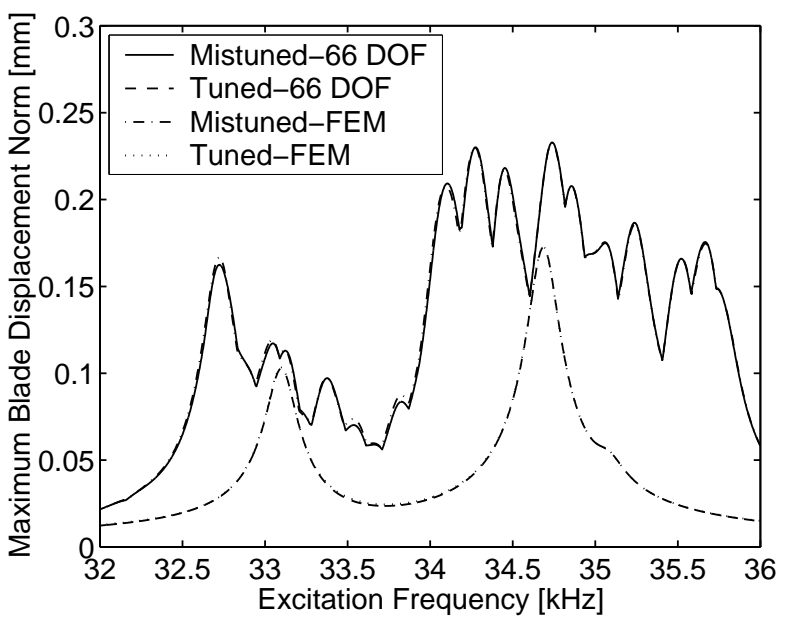

Fig. 14: Forced response for engine order 24 excitation, as obtained by the FEM and a 66-DOF CMM model

mal modes. Therefore, the number of mistuning patterns required is the number of cantilevered-blade normal modes to be used for the mistuning projection. Although the mode shapes of cantilevered blades change, eigenvalue mistuning values are sufficient to represent mistuning if the changes in mode shapes are negligible. Hence, Eq. (14) can be employed again, as in the proportional mistuning case.

The non-proportional mistuning is introduced by using two different sets of Young's moduli for the FEM of a cantilevered blade. One $\left(\delta_{n, 1}^{E}\right)$ is for the lower left and upper right parts of blades and the other $\left(\delta_{n, 2}^{E}\right)$ is for the lower right and upper left parts of blades. The Young's modulus mistuning parameters are listed in Table 2, and Fig. 15 shows the obtained eigenvalue mistuning patterns for several cantilevered-blade normal modes. 


\begin{tabular}{c|r|r||r|r|r}
\hline Blade & \multicolumn{1}{|c|}{$\delta_{n, 1}^{E}$} & \multicolumn{1}{c|}{$\delta_{n, 2}^{E}$} & Blade & \multicolumn{1}{|c}{$\delta_{n, 1}^{E}$} & \multicolumn{1}{c}{$\delta_{n, 2}^{E}$} \\
\hline 1 & 0.04080 & 0.01030 & 16 & 0.01990 & 0.03120 \\
2 & -0.06110 & -0.04990 & 17 & -0.02490 & -0.07530 \\
3 & 0.01430 & 0.02780 & 18 & 0.06380 & 0.01350 \\
4 & -0.06230 & -0.07580 & 19 & 0.03140 & -0.00080 \\
5 & -0.01170 & -0.00390 & 20 & -0.01220 & -0.00320 \\
6 & -0.02700 & -0.03210 & 21 & 0.03390 & -0.01210 \\
7 & 0.05190 & 0.00450 & 22 & -0.03220 & -0.04590 \\
8 & -0.06720 & -0.11630 & 23 & -0.00830 & 0.00530 \\
9 & 0.03710 & 0.01770 & 24 & 0.06010 & 0.08270 \\
10 & 0.06520 & 0.01460 & 25 & 0.02540 & 0.04540 \\
11 & 0.06790 & 0.05580 & 26 & -0.03980 & -0.08310 \\
12 & 0.04000 & 0.05910 & 27 & 0.04700 & 0.04230 \\
13 & -0.00850 & -0.05080 & 28 & 0.01780 & 0.01180 \\
14 & -0.00020 & -0.04850 & 29 & -0.05070 & -0.06600 \\
15 & -0.03960 & -0.02800 & & & \\
\hline
\end{tabular}

Table 2: Young's modulus mistuning parameters for the case study rotor

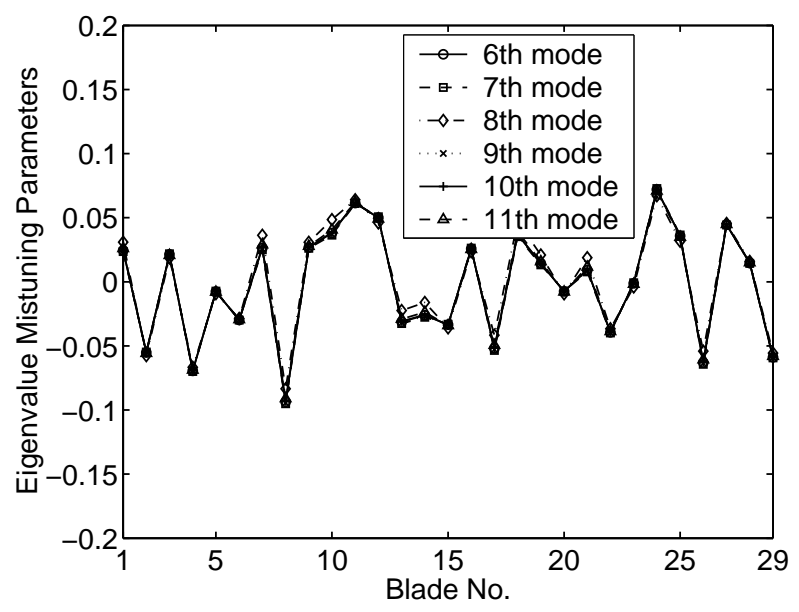

Fig. 15: Mistuning patterns of eigenvalues of cantilevered blades corresponding to the 7th through 11th normal mode

As can be seen in Fig. 15, the eigenvalue mistuning patterns are slightly different from each other. Thus, inaccurate results might be obtained by choosing one pattern among them, because the dynamic characteristics of a bladed disk are very sensitive to mistuning. Solution is to utilize all the mistuning patterns that are available. If there is only one blade-dominated mode family in the selected frequency band for a tuned-system normal mode basis, as in the case of the veering region 1 , a single mistuning pattern may be sufficient. However, if multiple blade-dominated mode families are close and they are included in a reduced-order model, as in the case of the veering region 2 , every dominant cantilevered-blade mode should have its own mistuning pattern.
The above comments are illustrated by investigating the forced response results for excitations corresponding to veering regions 1 and 2 . For veering region 1, only a single eigenvalue mistuning pattern corresponding to the 7th cantilevered-blade normal mode has a dominant effect on a reduced-order model as in the proportional mistuning case. For the reduced-order model by the CMM method, 106 tuned-system normal modes $(22-34 \mathrm{kHz})$ are employed for a basis. Figure 16 presents the forced responses for veering region 1 when mistuning is projected in two different ways. One way is to apply the 7th eigenvalue mistuning pattern to the 1st through 15th cantilevered-blade normal modes, which means that all the modes employed are mistuned by a single mistuning pattern. The other way is to apply the 6th through 8 th eigenvalue mistuning patterns to the corresponding modes, which means that the individual modes employed are mistuned differently. It is observed that individual mode mistuning does not improve the results. This shows again that the 7 th cantilevered-blade normal mode is dominant in the investigated frequency region, and, if there is only one blade-dominated mode group in the frequency range of interest, the response of a mistuned system can be predicted from a single eigenvalue mistuning pattern regardless of whether mistuning is proportional or not. The results by the CMM model are not as good as those for proportional mistuning in Fig. 12. This is because the changes in mode shapes are neglected and Eq. (14) is employed instead of Eq. (13). But, as shown in Fig. 16, the CMM model can find resonant frequencies and the peak values with small errors.

Figure 17 and 18 show the forced responses at 32$36 \mathrm{kHz}$ obtained by four different reduced-order models and by the FEM. As mentioned in the previous section, there are two blade-dominated mode groups in that range. In all four cases, 136 tuned-system normal modes $(26-43 \mathrm{kHz})$ are used as a basis for the reduced-order model. But, mistuning projection is performed differently. For Fig. 17 (a), a single mistuning pattern of the 8th cantilevered-blade normal mode is used, and for Fig. 17 (b), a single mistuning pattern of the 9th cantilevered-blade normal mode is used. In these single mistuning pattern cases, mistuning is projected to the lowest 15 cantilevered-blade normal modes. For Fig. 18 (a), the two mistuning patterns of the 8th and 9th cantilevered-blade normal modes are used to project the mistuning values to the corresponding cantileveredblade normal modes respectively. For Fig. 18 (b), the six mistuning patterns of the 6th to 11th cantilevered-blade normal modes are used for mistuning projection.

As these forced response results show clearly, the reduced-order models with two eigenvalue mistuning patterns and six eigenvalue mistuning patterns predict the behavior of the mistuned system better than the other 


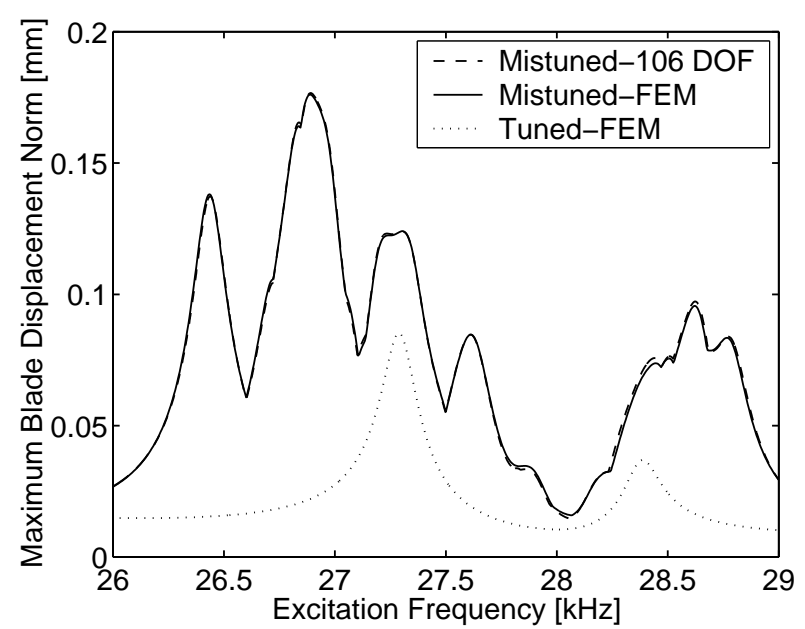

(a)

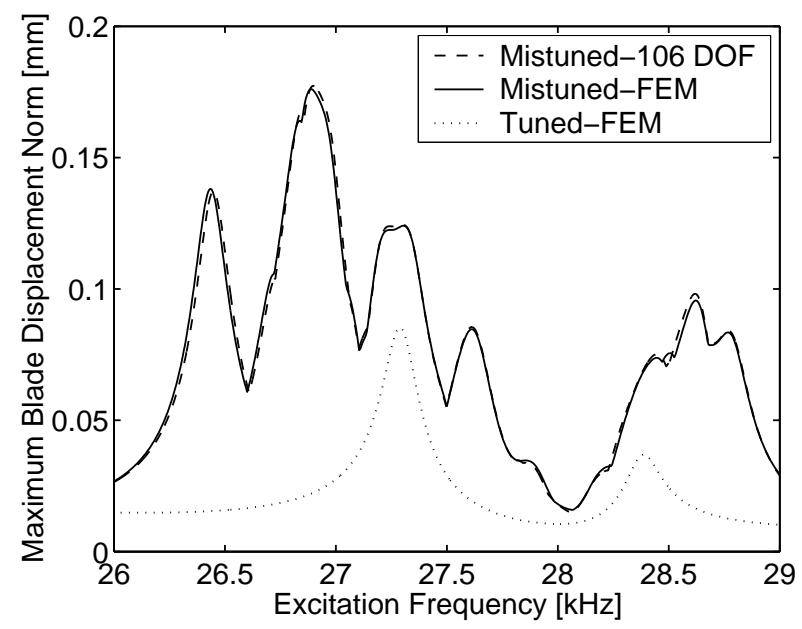

(b)

Fig. 16: Forced response for engine order 3 excitation, as obtained by the FEM and 106-DOF CMM models: (a) with a single eigenvalue mistuning pattern of the 7 th cantilevered-blade mode applied to the 1st through 15th cantilevered-blade normal modes, (b) with three eigenvalue mistuning patterns of the 6th to 8th cantilevered-blade normal mode applied to the corresponding cantilevered-blade normal modes

two cases. In fact, using a single mistuning pattern leads to poor results. This shows that, when multiple blade-dominated mode groups are so close that they interact in the response of a mistuned system, all the eigenvalue mistuning patterns corresponding to the dominant cantilevered-blade normal modes are required for a reduced-order model. When the dynamic behavior of a bladed disk with a few different eigenvalue mistuning patterns is to be predicted by a reduced-order model, it can be hard to estimate $\mathbf{K}^{\delta}$ in physical coordinates. Furthermore, if the changes in individual mistuned mode

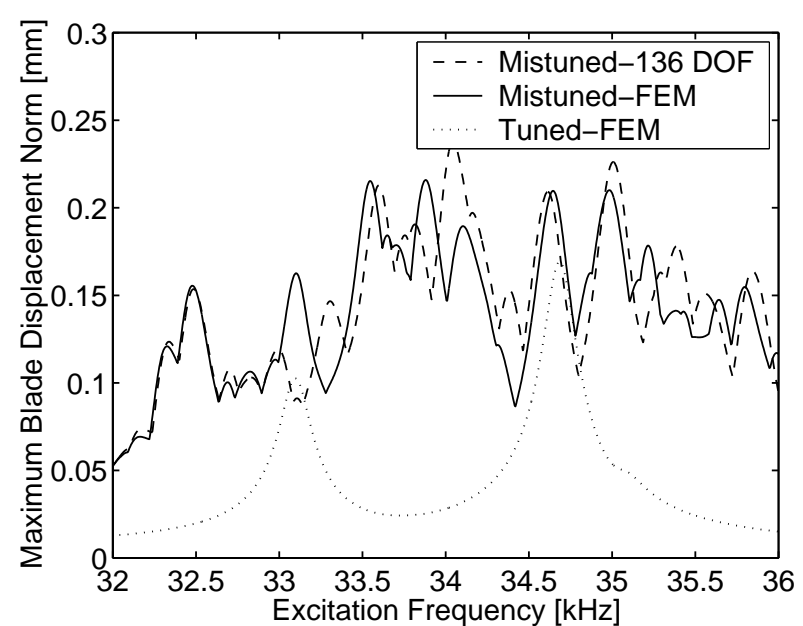

(a)

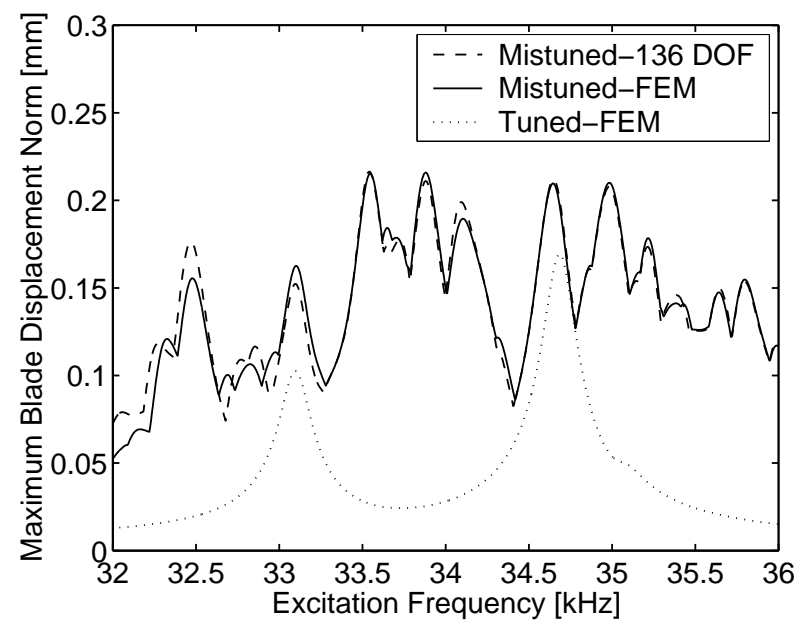

(b)

Fig. 17: Forced response for engine order 5 excitation, as obtained by the FEM and 136-DOF CMM models with a single mistuning pattern of (a) the 8th and (b) the 9th cantilevered-blade normal mode applied to the lowest 15 cantilevered-blade normal modes

shapes need to be considered, it is infeasible to estimate $\mathbf{K}^{\delta}$. Nevertheless, the CMM technique makes it easy to project non-proportional mistuning to tuned-system normal modes.

Figure 19 shows forced responses for the same frequency range as that in Fig. 18. The difference is that the change in mode shapes are considered and Eq. (13) is employed. The eigenvalues and mode shapes of the 1 st to 15 th cantilevered-blade normal modes are employed, and mistuning values corresponding to the $1 \mathrm{st}$ through 15th cantilevered-blade normal modes are obtained. Note that the eigenvalues and mode shapes are obtained from the finite element models of the mistuned blades in this study. For Fig. 19 (a), only the mistuning 


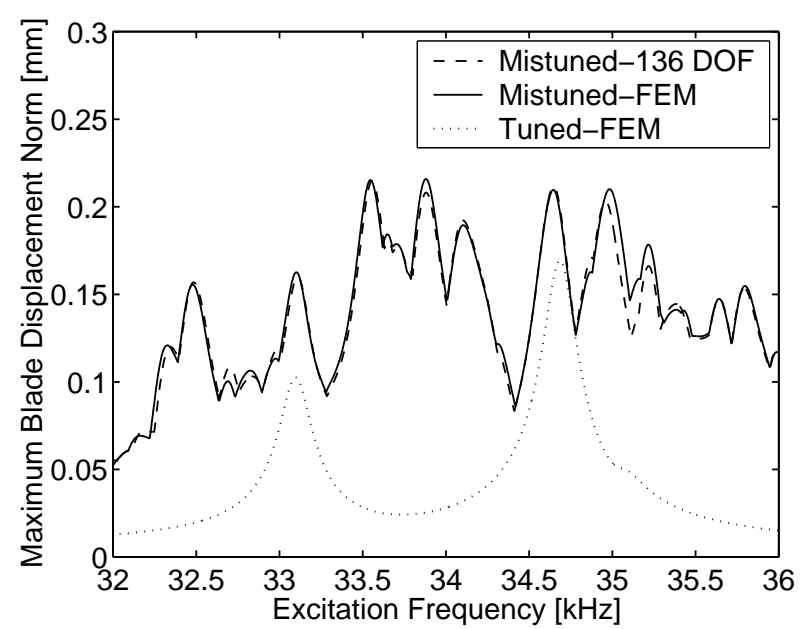

(a)

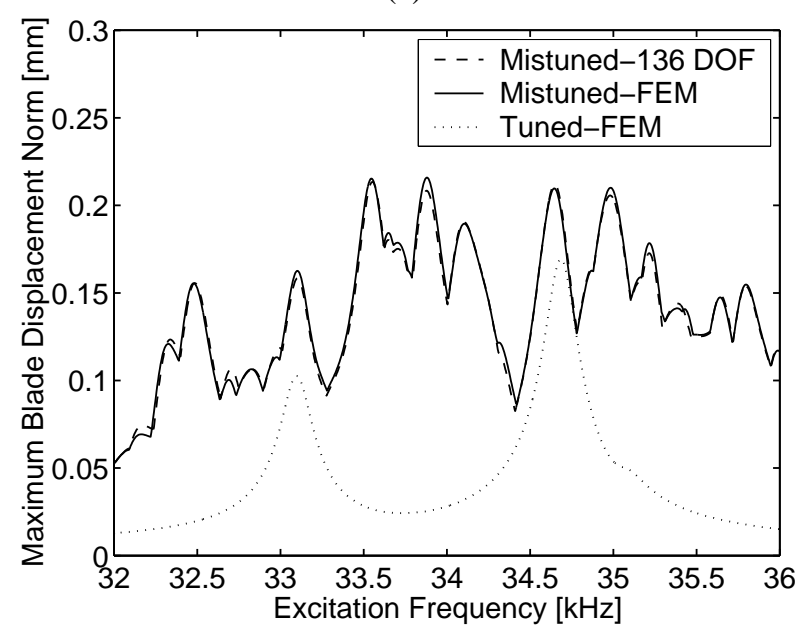

(b)

Fig. 18: Forced response for engine order 5 excitation, as obtained by the FEM and 136-DOF CMM models with eigenvalue mistuning patterns of: (a) the 8th and 9th cantilevered-blade normal modes applied to the corresponding cantilevered-blade normal modes, (b) the 6th to 11 th cantilevered-blade normal modes applied to the corresponding cantilevered-blade normal modes

values corresponding to the 8th and the 9th cantileveredblade normal modes are used in the reduced-order model. For Fig. 19 (b), the mistuning values corresponding to the 6 th and the 11th cantilevered-blade normal modes are used. As can be seen, these results show better accuracy than the results in Fig. 18 (c) and (d). This means that, when the change in mode shapes are considered, the accuracy can improves. Comparing Fig. 19 (a) and (b), the results in Fig. 19 (b) are slightly better. But, Fig. 19 (a) also shows excellent match between the results by the ROM and by the FEM. This implies that the small number of mistuning values lead to accurate results if the

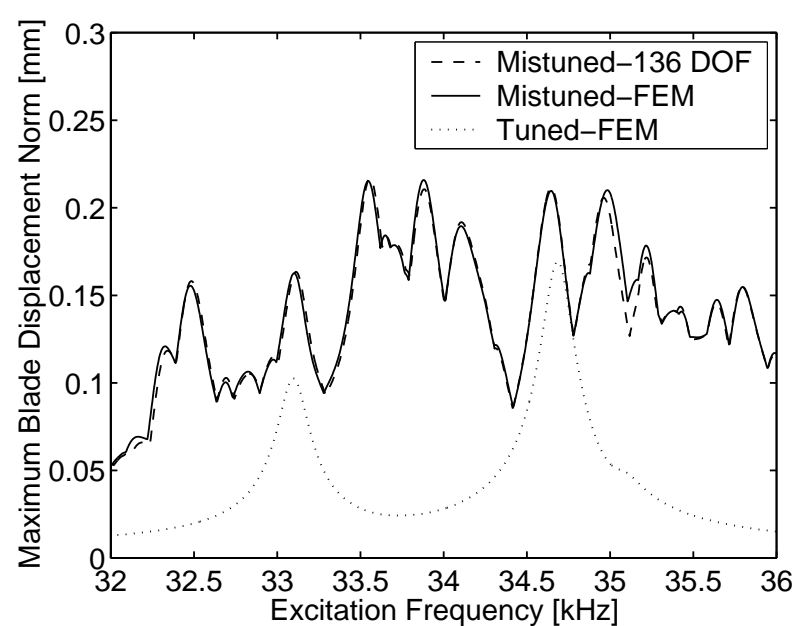

(a)

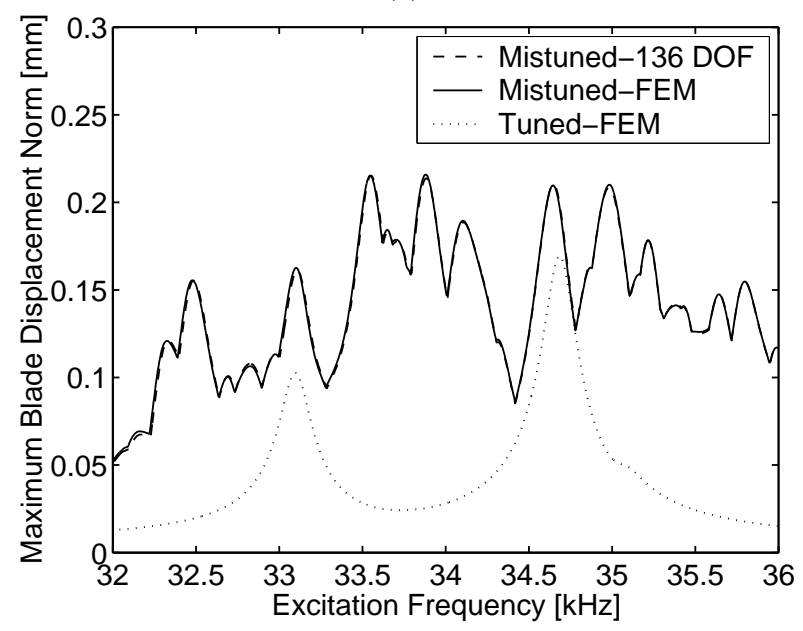

(b)

Fig. 19: Forced response for engine order 5 excitation, as obtained by the FEM and 136-DOF CMM models with mistuning values obtained using eigenvalues and mode shapes corresponding to: (a) the 8th and 9th cantileveredblade normal modes, and (b) the 6th to 11 th cantileveredblade normal modes

mistuning values are accurate. Therefore, when performing Monte Carlo simulations using the CMM method, a small number of random mistuning values are sufficient for the description of mistuning.

\subsection{Structural Interblade Coupling}

In this section, the modeling method presented in this paper is tested using a shrouded rotor. For a test case, the unshrouded rotor in Fig. 7 is modified to have shrouds so that the obtained shrouded rotor has the same geometry, constraints, and meshing, except for the added shrouds. One sector of the test case shrouded rotor and the shrouded blade are depicted in Fig. 20. Since the effect of shroud-to-shroud friction is not considered in 


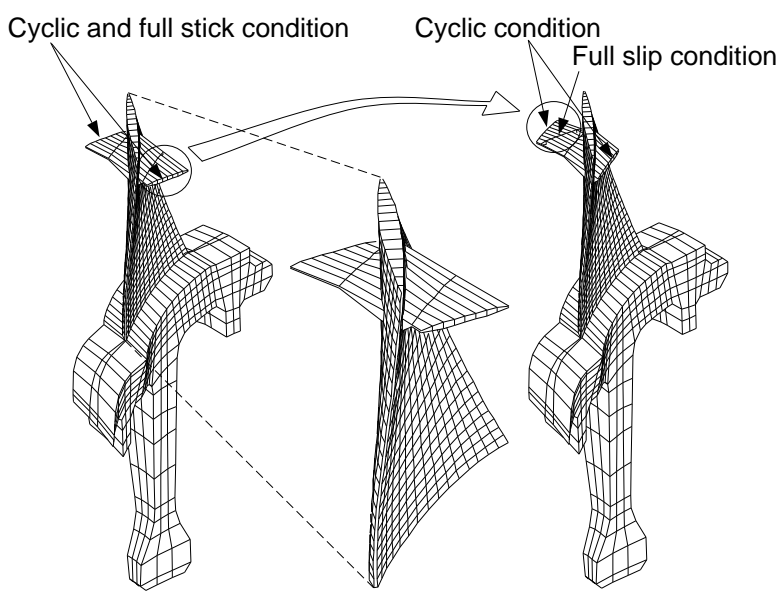

Fig. 20: Sector and blade models for the shrouded rotor

this study, the shroud boundary conditions are modeled as full stick or full slip.

In the same manner as in the previous unshrouded case, two finite element models are required to construct a CMM model: a complete one-sector model, and a blade-alone model with shrouds. For a one-sector model, cyclic constraints are applied to shroud-to-shroud interfaces as well as disk-to-disk interfaces. These cyclic constraints are easily implemented for the full-stick shroud boundary condition. However, for the full-slip boundary condition, it is impossible to apply the cyclic and slip conditions simultaneously to shroud interfaces. In order to solve this problem, the shroud boundary and cyclic boundary are separated as illustrated in Fig. 20. This configuration requires adding to the FEA output the deflections at the left-hand shroud piece of the right-hand adjacent sector, and replacing the output portion of the left-hand shroud piece of the original sector, because the mode shapes to be obtained from the one-sector model should be described by cantilevered-blade normal modes obtained from the shrouded blade-alone model.

The tuned-system natural frequencies versus number of nodal diameters are depicted in Fig. 21, as obtained from a finite element analysis of the one-sector models. Obviously, the steep lines to the left in Fig. 21 correspond to disk-dominated modes, as in the case of unshrouded rotors. However, the lines to corresponding to blade-dominated modes are not as horizontal as in the case of unshrouded rotors. This is because the blades are directly coupled by shrouds, and therefore, the dynamic stiffness increases as the number of nodal diameters increases. But, if the motion of a blade conforms to the motion of the shrouds, the effect of this stiffening can decrease. For example, the natural frequencies in the second torsional mode family (2T) of the full-stick shroud interface case in Fig. 21 decrease after nine nodal diam-

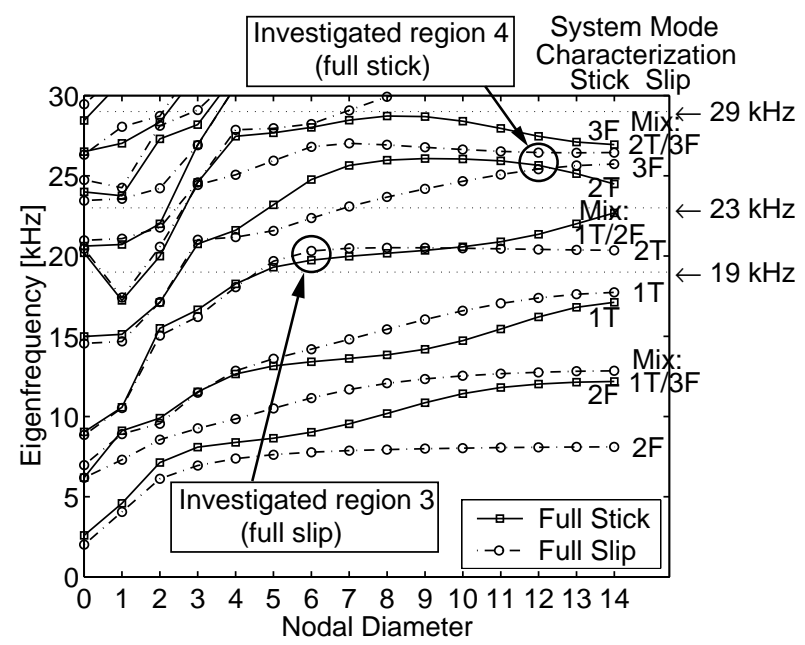

Fig. 21: Natural frequencies versus the number of nodal diameters for the shrouded tuned rotor FEM

eters. This stiffening effect makes it difficult to predict mistuned-system normal modes of a shrouded rotor relative to an unshrouded rotor because the modal density of a shrouded rotor is not as high. That is, since the modes are not closely spaced, more tuned-system normal modes are required to capture mistuned-system normal modes than for the case of a unshrouded rotor.

However, there still exist the regions of high modal density. Two regions are chosen to be studied; one is located at six nodal diameters around $20 \mathrm{kHz}$ for fullslip shrouds (region 3), and the other is located at twelve nodal diameters around $25.5 \mathrm{kHz}$ for full-stick shrouds (region 4). For these two regions, forced responses are obtained by CMM models and FEMs, and they are compared to validate CMM models.

For a blade-alone model, there are two kinds of structural boundaries. One is the disk-blade boundary and the other is a shroud-to-shroud boundary. The displacements at the disk-blade boundary are very small as in the case of the unshrouded model. But, the displacements at the shroud-to-shroud boundaries are not negligible. So, two different cantilevered blade models are available. One is a free-shroud model in which the disk-blade boundary is set to be fixed and the shroud-to-shroud boundaries are set to be free. The other is a fixed-shroud model in which all the boundaries are fixed.

A free-shroud model may not describe well the motion around the shroud-to-shroud boundaries, and the number of cantilevered-blade normal modes required to project mistuning can be more than that for a fixedshroud model. But, since the displacements at the diskblade boundary are very small, the effect of the mistuning corresponding to the boundary modes can be ignored, and normal-mode mistuning projection can be 


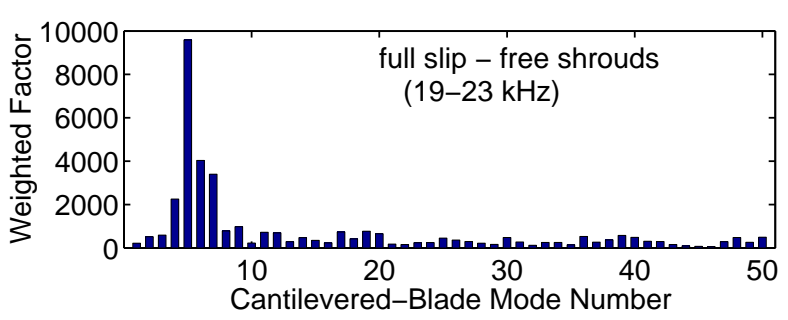

(a)

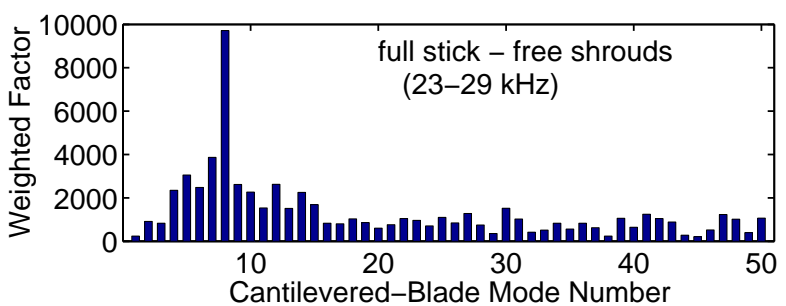

(b)

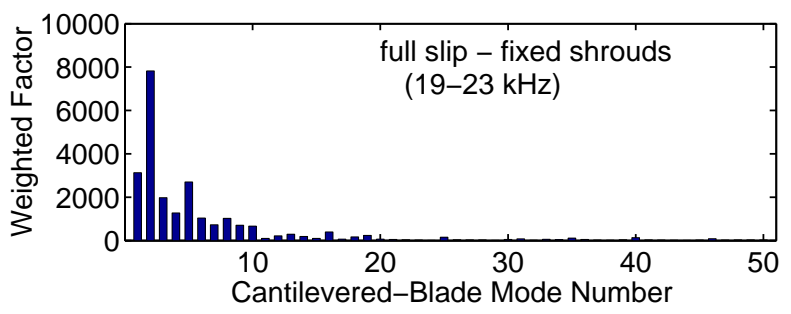

(c)

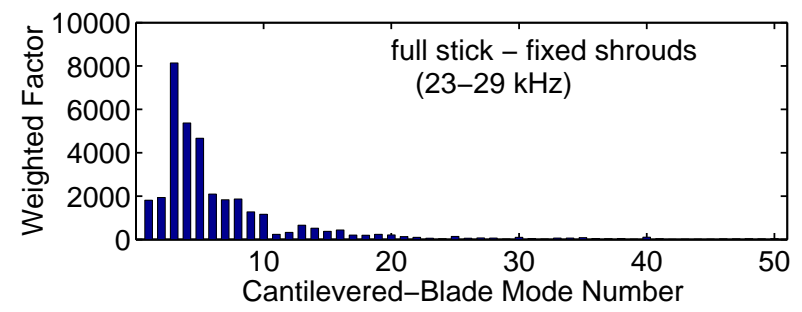

(d)

Fig. 22: Weighted average cantilevered-blade modal participation factors for the blade motion in the tunedsystem normal modes of the shrouded rotors with different shroud-to-shroud boundaries and blade-alone models

used. Therefore, Eq. (13) and (14) can be used for a freeshroud model, and individual mode mistuning is allowed.

A fixed-shroud model can describe better the blade motion. However, the boundary modes should be included for the mistuning projection in a CMM model, and only Eq. (12) is available. Since it is not practical to obtain the boundary mode mistuning values by measurements, actual non-proportional mistuning cannot be correctly implemented into an ROM. Nevertheless, if proportional mistuning is assumed, a fixed-shroud model will give better results than those of a free-shroud model. In order to compare these two kinds of blade-alone models with shrouds, proportional mistuning is introduced,

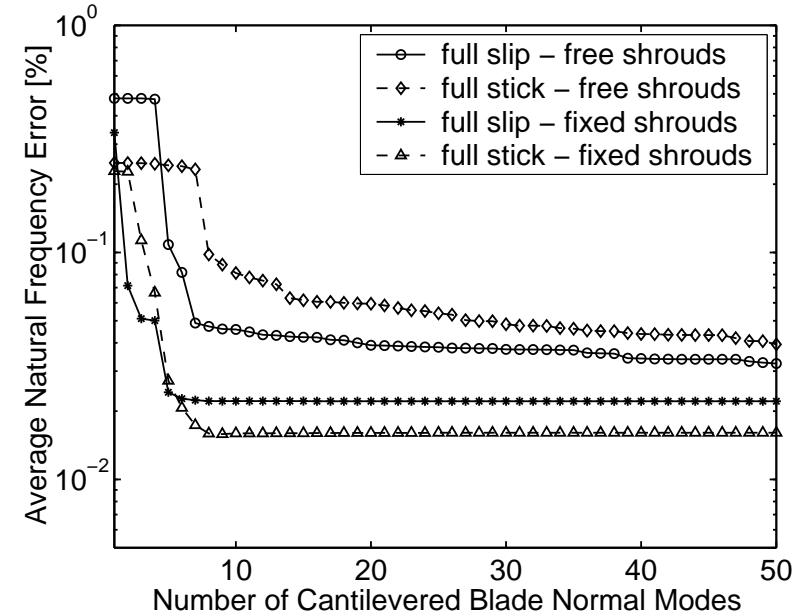

Fig. 23: Convergence of average eigenfrequency error for the shrouded rotor models with different shroud-toshroud boundary conditions, as obtained by using different blade-alone models

and the mistuning pattern in Table 1 is used.

For the mistuned-system normal modes around 20 $\mathrm{kHz}$ of the shrouded rotor with slip-conditioned shrouds, 36 tuned-system normal modes $(19-23 \mathrm{kHz})$ are chosen for a basis. For the mistuned-system normal modes around $25.5 \mathrm{kHz}$ of the shrouded rotor with stickconditioned shrouds, 59 tuned-system normal modes $(23-29 \mathrm{kHz})$ are chosen. Then, the modal participation factors of cantilevered-blade normal modes can be obtained using a free-shroud cantilevered blade and a fixedshroud cantilevered blade. The average modal participation factors weighted by the corresponding natural frequencies are displayed in Fig. 22. It can be seen that the modal participation factors of the higher modes in the free-shroud case (Fig. 22 (a) and (b)) are not as small as in the fixed-shroud case (Fig. 22 (c) and (d)). This is because more of the higher cantilevered-blade normal modes are required to describe shroud boundary motion. Yet, the lower modes are still more important.

The convergence of mistuned-system natural frequencies are tested by increasing the number of cantileveredblade normal modes used in the description of tunedsystem normal modes. The results are shown in Fig. 23. Both the free-shroud and the fixed-shroud models are tested with full-slip and full-stick boundary conditions. Only when the fixed-shroud model is employed, boundary mode mistuning projection is included. The fixedshroud model shows a fast convergence rate, while the convergence rate of the free-shroud model is slower after several modes are included.

For forced responses, unit loads are applied at the same locations as in the study of the previous unshrouded 


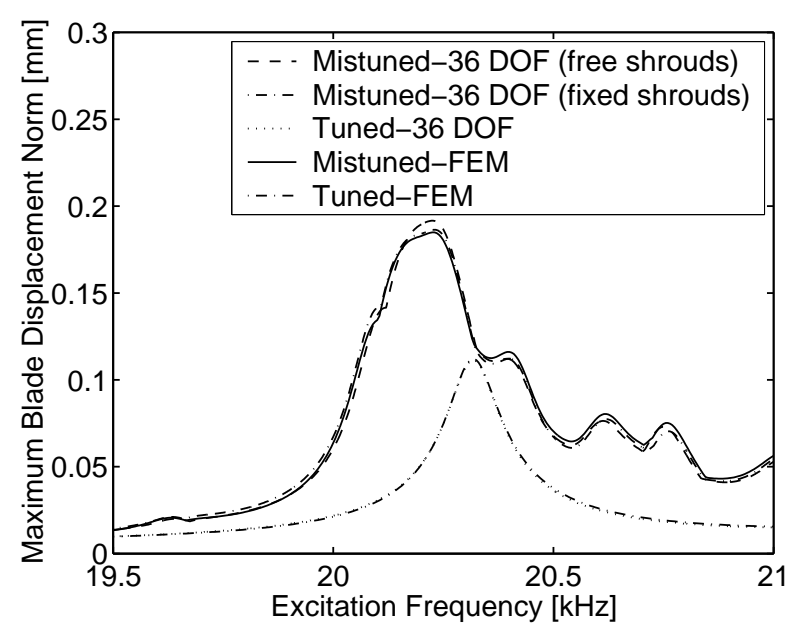

Fig. 24: Forced response for engine order 6 excitation, for both tuned and mistuned rotors with slip-conditioned shrouds, as obtained by the two different 36-DOF CMM models and by the FEM

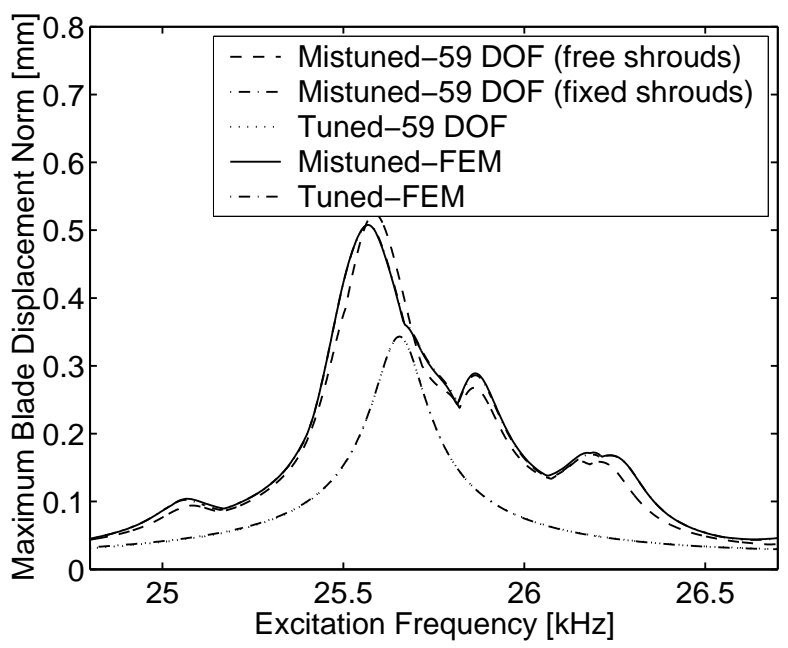

Fig. 25: Forced response for engine order 12 excitation, for both tuned and mistuned rotors with stickconditioned shrouds, as obtained by the two different 59DOF CMM models and by the FEM

rotor. Engine order 6 excitation is considered for the fullslip shroud case, and engine order 12 excitation is considered for the full-stick shroud case. For both cases, the lowest 15 cantilevered-blade normal modes are employed for normal mode mistuning projection. The resulting forced responses are shown in Fig. 24 and 25.

Although the results for the shrouded rotor are less accurate than those of the previous unshrouded rotor, the errors in resonant frequencies and magnitudes of responses are acceptable, considering that the modal density is not as high as that of an unshrouded rotor. As can be predicted from Fig. 23, the fixed-shroud model leads to obviously better results than does the free-shroud model. So, for the test-case shrouded rotor, restraining the shroud-to-shroud boundary and using boundary mode mistuning projection is the most appropriate approach. But, if mistuning is non-proportional, a freeshroud model should be considered.

\section{Conclusions}

A general reduced-order model (ROM) for a mistuned system has been developed by dividing a mistuned system into a tuned system and virtual mistuning components, regardless of the amount of mistuning. The model employs tuned-system normal modes and attachment modes to represent mistuned-system normal modes. From the general ROM, a compact ROM for a bladed disk with small blade mistuning has been also developed by the component mode mistuning (CMM) method, in which a mistuning projection is performed using the modal participation factors of cantilevered-blade component modes.

In the CMM method, the finite element models of a tuned sector and a tuned cantilevered blade are required. Cantilevered-blade normal modes and boundary modes are employed to describe the blade motion of the tunedsystem normal modes that are obtained from the onesector model. Thereby, mistuning values in the modal domain of the cantilevered-blade component modes are projected onto the tuned-system normal modes. Since the boundary modes in this paper are defined to minimize the corresponding modal mistuning values, the boundary modes can be neglected when the mistuning values in the elements near the boundaries are much smaller than those in the interior elements, or when the displacements at the boundaries in the tuned-system normal modes are much smaller than those at the interior part of a blade. Therefore, in many cases, modal mistuning values corresponding to cantilevered-blade normal modes are sufficient to predict the response of a mistuned system. This means that the implementation of actual arbitrary mistuning in an ROM is efficiently achieved. The modal mistuning values corresponding to cantileveredblade normal modes can be computed easily, relative to the mistuning values in the physical domain from the natural frequencies and mode shapes of mistuned blades, as demonstrated in section 3.2.

The modal participation factors of the cantileveredblade normal modes for the description of the blade motion in the tuned-system normal modes are also used for the implementation of aerodynamic coupling. Aerodynamic effects are represented by aerodynamic coefficients in complex cyclic modal coordinates of cantilevered-blade normal modes. Thereby, the aero- 
dynamic coefficients can be projected onto the tunedsystem normal modes.

The general ROM was validated using an industrial turbomachinery rotor with a rogue blade obtained by changing the blade geometry. It was observed that the estimated natural frequencies of the mistuned rotor converge rapidly as the selected number of tuned-system normal modes is increased. Also, the forced response results from the ROM (2,632 DOF) and the full finite element model $(126,846 \mathrm{DOF})$ show very good agreement.

The CMM method for small blade-mistuning was also validated using the same industrial rotor for proportional and non-proportional stiffness mistuning cases. By inspecting the modal participation factors, it was found that there are dominant cantilevered-blade modes that are sufficient for mistuning representation by themselves. For proportional mistuning, a single eigenvalue mistuning pattern was sufficient for mistuning implementation. For non-proportional mistuning, the mistuning values corresponding to individual dominant cantilevered-blade modes were required when two blade-dominated mode groups were close. Selecting just one eigenvalue mistuning pattern produced poor results.

Structural interblade coupling was investigated in the view of the application of the CMM method, using a shrouded rotor obtained by modifying the original industrial rotor in which proportional mistuning was introduced. The CMM models obtained from two different blade-alone models (free-shroud and fixed-shroud model) were compared. For the free-shroud model, boundary-mode mistuning was neglected, as in unshrouded rotor cases. For the fixed-shroud model, boundary-mode mistuning was included, and the effect of mistuning was captured better. However, it should be noted that, if mistuning is non-proportional, only the free-shroud model can be used.

\section{Acknowledgments}

This work is supported by the GUIde Consortium on blade durability at Carnegie Mellon University.

\section{References}

1 Wagner, J. T., "Coupling of Turbomachine Blade Vibrations Through the Rotor," ASME Journal of Engineering for Power, Vol. 89, No. 4, 1967, pp. 502-512.

2 Dye, R. C. F. and Henry, T. A., "Vibration Amplitudes of Compressor Blades Resulting From Scatter in Blade Natural Frequencies," ASME Journal of Engineering for Power, Vol. 91, No. 3, 1969, pp. 182188.
3 Ewins, D. J., "The Effects of Detuning Upon the Forced Vibrations of Bladed Disks," Journal of Sound and Vibration, Vol. 9, No. 1, 1969, pp. 65-79.

4 Ewins, D. J., "A Study of Resonance Coincidence in Bladed Discs," Journal Mechanical Engineering Science, Vol. 12, No. 5, 1970, pp. 305-312.

${ }^{5}$ El-Bayoumy, L. E. and Srinivasan, A. V., "Influence of Mistuning on Rotor-Blade Vibrations," AIAA Journal, Vol. 13, No. 4, 1975, pp. 460-464.

${ }^{6}$ Griffin, J. H. and Hoosac, T. M., "Model Development and Statistical Investigation of Turbine Blade Mistuning," ASME Journal of Vibration, Acoustics, Stress, and Reliability in Design, Vol. 106, 1984, pp. 204-210.

7 Wei, S. T. and Pierre, C., "Localization Phenomena in Mistuned Assemblies with Cyclic Symmetry, Part I: Free Vibrations," ASME Journal of Vibration, Acoustics, Stress, and Reliability in Design, Vol. 110, No. 4, 1988, pp. 429-438.

8 Wei, S. T. and Pierre, C., "Localization Phenomena in Mistuned Assemblies with Cyclic Symmetry, Part II: Forced Vibrations," ASME Journal of Vibration, Acoustics, Stress, and Reliability in Design, Vol. 110, No. 4, 1988, pp. 439-449.

9 Lin, C.-C. and Mignolet, M. P., "An Adaptive Perturbation Scheme for the Analysis of Mistuned Bladed Disks," ASME Journal of Engineering for Gas Turbines and Power, Vol. 119, No. 1, 1997, pp. 153-160.

10 Irretier, H., "Spectral Analysis of Mistuned Bladed Disk Assemblies by Component Mode Synthesis," in Vibrations of Bladed Disk Assemblies, Proceedings of the ASME 9th Biennial Conference on Mechanical Vibration and Noise, Dearborn, Michigan, 1983, pp. 115-125.

11 Kruse, M. J. and Pierre, C., "Forced Response of Mistuned Bladed Disks Using Reduced-Order Modeling," Proceedings of the 37th AIAA/ASME Structures, Structural Dynamics, and Materials Conference, Salt Lake City, Utah, 1996.

12 Kruse, M. J. and Pierre, C., "Dynamic Response of an Industrial Turbomachinery Rotor," Proceedings of the 32nd AIAA/ASME/SAE/ASEE Joint Propulsion Conference and Exhibit, Lake Buena Vista, Florida, 1996.

13 Castanier, M. P., Óttarsson, G., and Pierre, C., "A Reduced-Order Modeling Technique for Mistuned Bladed Disks," Journal of Vibration and Acoustics, Vol. 119, No. 3, 1997, pp. 439-447. 
14 Bladh, R., Castanier, M. P., and Pierre, C., "Reduced Order Modeling and Vibration Analysis of Mistuned Bladed Disk Assemblies with Shrouds," ASME Journal of Engineering for Gas Turbines and Power, Vol. 121, No. 3, 1999, pp. 515-522.

15 Bladh, R., Castanier, M. P., and Pierre, C., "Component-Mode-Based Reduced Order Modeling Techniques for Mistuned Bladed Disks-Part I: Theoretical Models," ASME Journal of Engineering for Gas Turbines and Power, Vol. 123, No. 1, 2001, pp. 89-99.

16 Bladh, R., Castanier, M. P., and Pierre, C., "Component-Mode-Based Reduced Order Modeling Techniques for Mistuned Bladed Disks-Part II: Application," ASME Journal of Engineering for Gas Turbines and Power, Vol. 123, No. 1, 2001, pp. 100108.

17 Moyroud, F., Jacquet-Richardet, G., and Fransson, T., "A Comparison of Two Finite Element Reduction Techniques for Mistuned Bladed-Disks," Proceedings of the 45th ASME Gas Turbine and Aeroengine Technical Congress, Exposition and Users Symposium, Munich, Germany, 2000.

18 Yang, M.-T. and Griffin, J. H., “A Reduced Order Approach for the Vibration of Mistuned Bladed Disk Assemblies," ASME Journal of Engineering for Gas Turbines and Power, Vol. 119, 1997, pp. 161-167.

19 Yang, M.-T. and Griffin, J. H., "A Reduced Order Model of Mistuning Using a Subset of Nominal System Modes," ASME Journal of Engineering for Gas Turbines and Power, Vol. 123, 2001, pp. 893-900.

20 Petrov, E. P., Sanliturk, K. Y., and Ewins, D. J., "A New Method for Dynamic Analysis of Mistuned Bladed Disks Based on the Exact Relationship Between Tuned and Mistuned Systems," ASME Journal of Engineering for Gas Turbines and Power, Vol. 124, 2002, pp. 586-597.

21 Pierre, C. and Murthy, D. V., "Aeroelastic Modal Characteristics of Mistuned Blade Assemblies: Mode Localization and Loss of Eigenstructure," AIAA Journal, Vol. 30, No. 10, 1992, pp. 2483-2496.

22 Yang, M.-T. and Griffin, J. H., “A Normalized Modal Eigenvalue Approach for Resolving Modal Interaction," ASME Journal of Engineering for Gas Turbines and Power, Vol. 119, 1997, pp. 647-650.

23 Bladh, R., Castanier, M. P., Pierre, C., and Kruse, M. J., "Dynamic Response Predictions for a Mistuned Industrial Turbomachinery Rotor Using Reduced Order Modeling," ASME Journal of Engineering for
Gas Turbines and Power, Vol. 124, No. 2, 2002, pp. 311-324.

\section{APPENDIX: Modal Participation Factors of Cantilevered-Blade Normal Modes}

Since a tuned bladed disk is a structure of cyclic symmetry, a full system mode can be represented by a mode for one sector in cyclic coordinates and its harmonic number. Therefore, once the modal participation factors for the blade portion of a cyclic one-sector mode are obtained, all the factors for the corresponding full system mode in physical coordinates can be easily computed.

Tuned-system normal modes can be obtained from a one-sector finite element model as

$$
\boldsymbol{\Phi}^{\mathrm{S}}=(\mathbf{F} \otimes \mathbf{I}) \underset{h=1, \ldots, P}{\tilde{\mathbf{B}} \operatorname{diag}}\left[\tilde{\boldsymbol{\Phi}}_{h}^{\mathrm{S}}\right]
$$

where $\mathbf{F}$ is a real Fourier matrix, $\underset{h=1, \ldots, P}{\tilde{\mathbf{B}} \operatorname{diag}[\cdot]}$ denotes a pseudo-block-diagonal matrix, and $\tilde{\boldsymbol{\Phi}}_{h}^{\mathrm{S}}$ is a real cyclic normal mode set corresponding to harmonic $h$. In this manner, the blade portion of tuned-system normal modes in physical coordinates is expressed as follows:

$$
\mathbf{\Phi}_{\Gamma}^{\mathrm{S}}=(\mathbf{F} \otimes \mathbf{I}) \underset{h=1, \ldots, P}{\tilde{\mathbf{B}} \operatorname{diag}}\left[\tilde{\boldsymbol{\Phi}}_{\Gamma, h}^{\mathrm{S}}\right]
$$

Now, $\tilde{\boldsymbol{\Phi}}_{\Gamma, h}^{\mathrm{S}}$ is described by cantilevered-blade component modes.

$$
\tilde{\boldsymbol{\Phi}}_{\Gamma, h}^{\mathrm{S}}=\left[\begin{array}{c}
\tilde{\boldsymbol{\Phi}}_{\mathrm{i}, \Gamma, h}^{\mathrm{S}} \\
\tilde{\boldsymbol{\Phi}}_{\mathrm{b}, \Gamma, h}^{\mathrm{S}}
\end{array}\right]=\left\{\begin{array}{l}
{\left[\begin{array}{cc}
\boldsymbol{\Phi}^{\mathrm{CB}} & \mathbf{\Psi}^{\mathrm{CB}, m} \\
\mathbf{0} & \mathbf{I}
\end{array}\right]\left[\begin{array}{l}
\tilde{\mathbf{q}}_{\phi, h}^{\mathrm{CB}, m} \\
\tilde{\mathbf{q}}_{\psi, h}^{\mathrm{CB}, m}
\end{array}\right]} \\
{\left[\begin{array}{cc}
\boldsymbol{\Phi}^{\mathrm{CB}} & \boldsymbol{\Psi}^{\mathrm{CB}, k} \\
\mathbf{0} & \mathbf{I}
\end{array}\right]\left[\begin{array}{l}
\tilde{\mathbf{q}}_{\phi, h}^{\mathrm{CB}, k} \\
\tilde{\mathbf{q}}_{\psi, h}^{\mathrm{CB}, k}
\end{array}\right],}
\end{array}\right.
$$

where $\tilde{\boldsymbol{\Phi}}_{\mathrm{i}, \Gamma, h}^{\mathrm{S}}$ and $\tilde{\boldsymbol{\Phi}}_{\mathrm{b}, \Gamma, h}^{\mathrm{S}}$ correspond to the interior DOF and the boundary DOF of a cantilevered blade of which the boundary is fixed, and $\tilde{\mathbf{q}}_{\phi, h}^{\mathrm{CB}, m}, \tilde{\mathbf{q}}_{\psi, h}^{\mathrm{CB}, m}, \tilde{\mathbf{q}}_{\phi, h}^{\mathrm{CB}, k}$, and $\tilde{\mathbf{q}}_{\psi, h}^{\mathrm{CB}, k}$ correspond to participation factors of cantileveredblade normal and boundary modes in the blade portion of cyclic tuned-system normal modes of harmonic $h$. From Eq. (24), it is obvious that

$$
\tilde{\mathbf{q}}_{\psi, h}^{\mathrm{CB}, m}=\tilde{\mathbf{q}}_{\psi, h}^{\mathrm{CB}, k}=\tilde{\mathbf{\Phi}}_{\mathrm{b}, \Gamma, h}^{\mathrm{S}} .
$$

Before calculating the modal participation factors of cantilevered-blade normal modes, it should be noted that the normal modes and the boundary modes of a cantilevered blade are orthogonal with respect to nominal 
mass and stiffness matrices. For the proof, using Eq. (9),

$$
\begin{aligned}
& {\left[\begin{array}{c}
\boldsymbol{\Phi}^{\mathrm{CB}} \\
\mathbf{0}
\end{array}\right]^{T}\left[\begin{array}{cc}
\mathbf{M}_{\mathrm{ii}}^{\mathrm{CB}} & \mathbf{M}_{\mathrm{ib}}^{\mathrm{CB}} \\
\mathbf{M}_{\mathrm{ib}}^{\mathrm{CB}} & \mathbf{M}_{\mathrm{bb}}^{\mathrm{CB}}
\end{array}\right]\left[\begin{array}{c}
\boldsymbol{\Psi}^{\mathrm{CB}, m} \\
\mathbf{I}
\end{array}\right]} \\
& =\left[\begin{array}{c}
\boldsymbol{\Phi}^{\mathrm{CB}} \\
\mathbf{0}
\end{array}\right]^{T}\left[\begin{array}{c}
\mathbf{M}_{\mathrm{ii}}^{\mathrm{CB}} \mathbf{\Psi}^{\mathrm{CB}, m}+\mathbf{M}_{\mathrm{ib}}^{\mathrm{CB}} \\
\mathbf{M}_{\mathrm{ib}}^{\mathrm{CB}} \boldsymbol{\Psi}^{\mathrm{CB}, m}+\mathbf{M}_{\mathrm{bb}}^{\mathrm{CB}}
\end{array}\right] \\
& =\left[\begin{array}{c}
\boldsymbol{\Phi}^{\mathrm{CB}} \\
\mathbf{0}
\end{array}\right]^{T}\left[\begin{array}{c}
\mathbf{0} \\
\mathbf{M}_{\mathrm{ib}}^{\mathrm{CB}}{ }^{T} \mathbf{\Psi}^{\mathrm{CB}, m}+\mathbf{M}_{\mathrm{bb}}^{\mathrm{CB}}
\end{array}\right]=\mathbf{0} \\
& {\left[\begin{array}{c}
\boldsymbol{\Phi}^{\mathrm{CB}} \\
\mathbf{0}
\end{array}\right]^{T}\left[\begin{array}{cc}
\mathbf{K}_{\mathrm{ii}}^{\mathrm{CB}} & \mathbf{K}_{\mathrm{ib}}^{\mathrm{CB}} \\
\mathbf{K}_{\mathrm{ib}}^{\mathrm{CB} T} & \mathbf{K}_{\mathrm{bb}}^{\mathrm{CB}}
\end{array}\right]\left[\begin{array}{c}
\boldsymbol{\Psi}^{\mathrm{CB}, k} \\
\mathbf{I}
\end{array}\right]} \\
& =\left[\begin{array}{c}
\boldsymbol{\Phi}^{\mathrm{CB}} \\
\mathbf{0}
\end{array}\right]^{T}\left[\begin{array}{c}
\mathbf{K}_{\mathrm{ii}}^{\mathrm{CB}} \mathbf{\Psi}^{\mathrm{CB}, k}+\mathbf{K}_{\mathrm{ib}}^{\mathrm{CB}} \\
\mathbf{K}_{\mathrm{ib}}^{\mathrm{CB}}{ }^{T} \boldsymbol{\Psi}^{\mathrm{CB}, k}+\mathbf{K}_{\mathrm{bb}}^{\mathrm{CB}}
\end{array}\right] \\
& =\left[\begin{array}{c}
\boldsymbol{\Phi}^{\mathrm{CB}} \\
\mathbf{0}
\end{array}\right]^{T}\left[\begin{array}{c}
\mathbf{0} \\
\mathbf{K}_{\mathrm{ib}}^{\mathrm{CB}}{ }^{T} \mathbf{\Psi}^{\mathrm{CB}, k}+\mathbf{K}_{\mathrm{bb}}^{\mathrm{CB}}
\end{array}\right]=\mathbf{0}
\end{aligned}
$$

Using these orthogonality conditions, $\tilde{\mathbf{q}}_{\phi, h}^{\mathrm{CB}, m}$ and $\tilde{\mathbf{q}}_{\phi, h}^{\mathrm{CB}, k}$ can be obtained from Eq. (24) as follows:

$$
\begin{aligned}
& {\left[\begin{array}{c}
\boldsymbol{\Phi}^{\mathbf{C B}} \\
\mathbf{0}
\end{array}\right]^{T} \mathbf{M}^{\mathrm{CB}} \tilde{\boldsymbol{\Phi}}_{\Gamma, h}^{\mathrm{S}}} \\
& =\left[\begin{array}{c}
\boldsymbol{\Phi}^{\mathrm{CB}} \\
\mathbf{0}
\end{array}\right]^{T} \mathbf{M}^{\mathrm{CB}}\left[\begin{array}{cc}
\boldsymbol{\Phi}^{\mathrm{CB}} & \mathbf{\Psi}^{\mathrm{CB}, m} \\
\mathbf{0} & \mathbf{I}
\end{array}\right]\left[\begin{array}{l}
\tilde{\mathbf{q}}_{\phi, h}^{\mathrm{CB}, m} \\
\tilde{\mathbf{q}}_{\psi, h}^{\mathrm{CB}, m}
\end{array}\right] \\
& =\left[\begin{array}{ll}
\mathbf{I} & \mathbf{0}
\end{array}\right]\left[\begin{array}{l}
\tilde{\mathbf{q}}_{\phi, h}^{\mathrm{CB}, m} \\
\tilde{\mathbf{q}}_{\psi, h}^{\mathrm{CB}, m}
\end{array}\right]=\tilde{\mathbf{q}}_{\phi, h}^{\mathrm{CB}, m} \\
& {\left[\begin{array}{c}
\boldsymbol{\Phi}^{\mathrm{CB}} \\
\mathbf{0}
\end{array}\right]^{T} \mathbf{K}^{\mathrm{CB}} \tilde{\boldsymbol{\Phi}}_{\Gamma, h}^{\mathrm{S}}} \\
& =\left[\begin{array}{c}
\boldsymbol{\Phi}^{\mathrm{CB}} \\
\mathbf{0}
\end{array}\right]^{T} \mathbf{K}^{\mathrm{CB}}\left[\begin{array}{cc}
\boldsymbol{\Phi}^{\mathrm{CB}} & \mathbf{\Psi}^{\mathrm{CB}, k} \\
\mathbf{0} & \mathbf{I}
\end{array}\right]\left[\begin{array}{l}
\tilde{\mathbf{q}}_{\phi, h}^{\mathrm{CB}, k} \\
\tilde{\mathbf{q}}_{\psi, h}^{\mathrm{CB}, k}
\end{array}\right] \\
& =\left[\begin{array}{ll}
\boldsymbol{\Lambda}^{\mathrm{CB}} & \mathbf{0}
\end{array}\right]\left[\begin{array}{l}
\tilde{\mathbf{q}}_{\phi, h}^{\mathrm{CB}, k} \\
\tilde{\mathbf{q}}_{\psi, h}^{\mathrm{CB}, h}
\end{array}\right]=\boldsymbol{\Lambda}^{\mathrm{CB}} \tilde{\mathbf{q}}_{\phi, h}^{\mathrm{CB}, k}
\end{aligned}
$$

Once $\tilde{\mathbf{q}}_{\phi, h}^{\mathrm{CB}, m}$ and $\tilde{\mathbf{q}}_{\phi, h}^{\mathrm{CB}, k}$ are obtained, $\mathbf{q}_{\phi, n}^{\mathrm{CB}, m}$ and $\mathbf{q}_{\phi, n}^{\mathrm{CB}, k}$ are expressed using the real Fourier matrix, F, and Kronecker product in the same manner as in Eq. (22) and (23). That is,

$$
\begin{gathered}
{\left[\begin{array}{c}
\vdots \\
\mathbf{q}_{\phi, n}^{\mathrm{CB}, m} \\
\vdots
\end{array}\right]=(\mathbf{F} \otimes \mathbf{I}) \underset{h=1, \ldots, P}{\tilde{\mathbf{B}} \operatorname{diag}}\left[\tilde{\mathbf{q}}_{\phi, h}^{\mathrm{CB}, m}\right]} \\
{\left[\begin{array}{c}
\vdots \\
\mathbf{q}_{\phi, n}^{\mathrm{CB}, k} \\
\vdots
\end{array}\right]=(\mathbf{F} \otimes \mathbf{I}) \underset{h=1, \ldots, P}{\tilde{\mathbf{B}} \operatorname{diag}}\left[\tilde{\mathbf{q}}_{\phi, h}^{\mathrm{CB}, k}\right]}
\end{gathered}
$$

